\title{
THE DEVELOPMENT OF TRAVELLING WAVES IN QUADRATIC AND CUBIC AUTOCATALYSIS WITH UNEQUAL DIFFUSION RATES. III: LARGE TIME DEVELOPMENT IN QUADRATIC AUTOCATALYSIS
}

\author{
BY \\ J. BILLINGHAM AND D. J. NEEDHAM \\ University of East Anglia, Norwich, U.K.
}

\begin{abstract}
We study the isothermal, quadratic autocatalytic reaction scheme $A+$ $B \rightarrow 2 B$, where $A$ is a reactant and $B$ is an autocatalyst. We consider the situation when a quantity of $B$ is introduced locally into a uniform expanse of $A$ in onedimensional slab geometry. By analysing the large time asymptotic solution of the resulting initial value problem we show that either a permanent form travelling wave or a phase wave develops. In the case when a travelling wave evolves we determine the first two terms in the large time asymptotic expansion of the propagation speed, which is dependent upon the asymptotic form of the initial input distribution of $B$ far ahead of the wavefront.
\end{abstract}

1. Introduction. In this paper, we consider the isothermal, quadratic autocatalytic chemical reaction scheme,

$$
A+B \rightarrow 2 B, \quad \text { rate } k a b,
$$

where $a$ and $b$ are concentrations of the reactant, $A$, and the autocatalyst, $B$, respectively, and $k$ is a reaction rate constant. The reaction step (1) has been used in several successful models of real chemical systems, which include the BelousovZhabotinskii reaction [5] and also gas-phase, radical chain-branching, oxidation reactions, such as the carbon monoxide-oxygen and hydrogen-oxygen systems [7, 13]. Autocatalytic reaction rate laws also arise in enzyme reactions such as glycolysis [14].

Observations show that chemical systems for which quadratic autocatalysis forms a key step can support propagating chemical wavefronts when the reaction mixture is unstirred $[8,16]$. These wavefronts, or travelling waves, arise via a combination of reaction and diffusion. Physically, the typical situation that leads to the development of travelling waves is that which arises when a quantity of the autocatalyst, $B$, is introduced locally into an expanse of the reactant, $A$, which is initially at uniform concentration. The developing reaction is often observed to generate wavefronts, which propagate outward from the initial reaction zone. It is this phenomenon that

Received December 4, 1990.

First author's current address: Schumberger Cambridge Research Ltd., P.O. Box 153, Cambridge, CB3 OHG, England. 
we study in this paper. In order to simplify the analysis, we restrict attention to the case of one-dimensional slab geometry, with the coordinate $\bar{x}$ measuring distance.

The equations that govern the reaction and diffusion of $A$ and $B$ under reaction scheme (1) are

$$
\begin{aligned}
& \frac{\partial a}{\partial \bar{t}}=D_{A} \frac{\partial^{2} a}{\partial \bar{x}^{2}}-k a b, \\
& \frac{\partial b}{\partial \bar{t}}=D_{B} \frac{\partial^{2} b}{\partial \bar{x}^{2}}+k a b .
\end{aligned}
$$

Here, $\bar{t}$ is time, and $D_{A}$ and $D_{B}$ are the constant diffusion rates of the reactant, $A$, and the autocatalyst, $B$, respectively. The initial conditions are

$$
a(\bar{x}, 0)=a_{0}, \quad b(\bar{x}, 0)=b_{0} g(\bar{x}), \quad|\bar{x}|<\infty,
$$

where $g(\bar{x})$ is a given nonnegative function of $\bar{x}$ with a maximum value of unity and $g(\bar{x}) \rightarrow 0$ as $|\bar{x}| \rightarrow \infty$. Here, $a_{0}$ and $b_{0}$ are the positive, constant initial concentration of $A$ and maximum initial concentration of $B$, respectively. The boundary conditions to be satisfied by $a$ and $b$ are

$$
a(\bar{x}, \bar{t}) \rightarrow a_{0}, \quad b(\bar{x}, \bar{t}) \rightarrow 0, \quad|\bar{x}| \rightarrow \infty, \bar{t} \geq 0 .
$$

For the purpose of this paper, it is also convenient to consider $g(\bar{x})$ as symmetric about the point $\bar{x}=0$. The problem can then be reduced to the domain $\bar{x} \geq 0$ with a symmetry condition at $\bar{x}=0$. After making this additional assumption, which is not at all restrictive, the initial and boundary conditions $(3 a, b)$ become

$$
\begin{gathered}
a(\bar{x}, 0)=a_{0}, \quad b(\bar{x}, 0)=b_{0} g(\bar{x}), \quad \bar{x} \geq 0 ; \\
\frac{\partial a}{\partial \bar{x}}(0, \bar{t})=\frac{\partial b}{\partial \bar{x}}(0, \bar{t})=0, \quad \bar{t} \geq 0 ; \\
a(\bar{x}, \bar{t}) \rightarrow a_{0}, \quad b(\bar{x}, \bar{t}) \rightarrow 0 \quad \text { as } \bar{x} \rightarrow \infty, \quad \bar{t} \geq 0 .
\end{gathered}
$$

The initial value problem given by Eq. (2), together with initial and boundary conditions (4), is also appropriate to model the situation where the reaction proceeds on the domain $\bar{x} \geq 0$ with an impermeable barrier positioned at $\bar{x}=0$.

It is now convenient to introduce dimensionless variables as

$$
\alpha=a / a_{0}, \quad \beta=b / a_{0}, \quad t=k a_{0} \bar{t}, \quad x=\left(k a_{0} / D_{A}\right)^{1 / 2} \bar{x},
$$

in terms of which Eqs. (2), together with initial and boundary conditions (4), become

$$
\begin{aligned}
& \frac{\partial \alpha}{\partial t}=\frac{\partial^{2} \alpha}{\partial x^{2}}-\alpha \beta, \\
& \frac{\partial \beta}{\partial t}=D \frac{\partial^{2} \beta}{\partial x^{2}}+\alpha \beta,
\end{aligned}
$$

where $D=D_{B} / D_{A}$ and

$$
\begin{gathered}
\alpha(x, 0)=1, \quad \beta(x, 0)=\beta_{0} g(x), \quad x \geq 0 ; \\
\frac{\partial \alpha}{\partial x}(0, t)=\frac{\partial \beta}{\partial x}(0, t)=0, \quad t \geq 0 ; \\
\alpha(x, t) \rightarrow 1, \quad \beta(x, t) \rightarrow 0 \quad \text { as } x \rightarrow \infty, \quad t \geq 0 .
\end{gathered}
$$


The dimensionless parameter $\beta_{0}=b_{0} / a_{0}$ provides a measure of the maximum concentration of the initial input of the autocatalyst, whilst the dimensionless parameter $D$ measures the rate of diffusion of the autocatalyst, $B$, relative to that of the reactant, $A$. In chemical systems that involve reactants of similar molecular weight it is a reasonable approximation to assume that the diffusion rates, $D_{A}$ and $D_{B}$, are equal, which gives $D=1$. However, enzyme reactions may involve large enzyme molecules and much smaller substrate molecules, which leads to significantly different diffusion rates. Equations (6) also arise in epidemiology, where $\alpha$ represents the number density of healthy individuals [1]. Infected individuals may be significantly more or less mobile than healthy individuals. This situation again leads to a value for $D$, which can be well away from unity. The interesting case $D=0$, which models the situation when the autocatalyst, $B$, is immobilized, was studied in part II of this series of papers [3]. In this paper we consider $D>0$.

When $D=1$, we can add Eq. (6a) and (6b) to obtain a diffusion equation that has the solution,

$$
\alpha+\beta=1+\frac{\beta_{0}}{2(\pi t)^{1 / 2}} \int_{-\infty}^{\infty} g(|\xi|) e^{-(x-\xi)^{2} / 4 t} d \xi,
$$

which satisfies the initial and boundary conditions (7a, b, c). Therefore, if $\int_{0}^{\infty} g(x) d x$ exists, $\alpha+\beta \rightarrow 1$ as $t \rightarrow \infty$, uniformly for all $x \geq 0$. The large time asymptotic solution of the initial value problem (6), (7) is therefore closely related to the large time asymptotic solution of the initial value problem,

$$
\begin{gathered}
\frac{\partial \beta}{\partial t}=\frac{\partial^{2} \beta}{\partial x^{2}}+\beta(1-\beta) ; \\
\beta(x, 0)=\beta_{0} g(x), \quad x \geq 0 ; \\
\frac{\partial \beta}{\partial x}(0, t)=0, \quad t \geq 0 ; \\
\beta(x, t) \rightarrow 0 \text { as } t \rightarrow \infty, \quad t \geq 0 .
\end{gathered}
$$

This problem has a long history and was originally proposed by Fisher [6] as a model for the advance of an advantageous gene. Fisher showed that Eq. (9a) has a permanent form travelling wave solution with constant propagation speed $\hat{v}$ for each $\hat{v} \geq 2$. A permanent form travelling wave solution is a nontrivial, nonnegative solution which depends on the single variable $z \equiv x-\hat{v} t$ and satisfies the conditions $\beta \rightarrow 0$ as $z \rightarrow \infty$ and $\beta \rightarrow 1$ as $z \rightarrow-\infty$. Kolmogorov, Petrovsky, and Piskounov [9] showed that for a step function initial distribution of $\beta$, the solution evolves into a permanent form travelling wave with propagation speed $v(t) \rightarrow 2$ as $t \rightarrow \infty$. McKean [11] exploited the fact that the maximal displacement of branching Brownian motion satisfies equation (9a) to derive the result of Kolmogorov et al. and also to show that if $g(x)=O\left(e^{-\sigma x}\right)$ as $x \rightarrow \infty$, a permanent form travelling wave evolves, with propagation speed $v(t) \rightarrow(\sigma+1 / \sigma)$ for $0<\sigma \leq 1$ and $v(t) \rightarrow 2$ for $\sigma \geq 1$, as $t \rightarrow \infty$. These results were also obtained by Larson [10] who used comparison theorems to obtain upper and lower bounds on the solution of the initial value problem (9). In addition, Larson showed that if $e^{\sigma x} g(x) \rightarrow \infty$ as $x \rightarrow \infty$ for 
all $\sigma>0$, no travelling wave evolves. Bramson [4] extended the work of McKean to show that for a step function initial distribution of $\beta$, the travelling wave propagation speed $v(t) \sim 2-\frac{3}{2} t^{-1}$ as $t \rightarrow \infty$. This result was also obtained formally, by Merkin and Needham [12], using matched asymptotic expansions to obtain the large time solution of the related initial value problem (6), (7) with $D=1$. In part I of this series of papers [2] we studied the permanent form travelling wave solutions of Eq. (6) and showed that these exist for each $\hat{v} \geq 2 \sqrt{D}$. In [3] we obtained the asymptotic solution of the initial value problem (6), (7) for $0<D \ll 1$ when the initial input function $g(x)$ has compact support $(g(x) \equiv 0$ for $x>\lambda>0)$. In this case, a travelling wave evolves and propagates out of the initial reaction zone with the minimum speed, $2 \sqrt{D}$. In this paper, we extend the work of Merkin and Needham [12] by using matched asymptotic expansions to obtain the solution of the initial value problem (6), (7) for $t \gg 1$ with $D>0$. In addition, we no longer restrict attention to the case when $g(x)$ has compact support but consider various classes of function $g(x)$. Analysis of this asymptotic solution then leads to the following conclusions.

Result 1.1. If $\{\alpha(x, t), \beta(x, t)\}$ is the solution of the initial value problem (6), (7) and

(a) $e^{\sigma x} g(x) \rightarrow \infty$ as $x \rightarrow \infty$ for all $\sigma>0$, no permanent form travelling wave evolves, in the sense that $\alpha(\tilde{z}+c t, t) \rightarrow 0, \beta(\tilde{z}+c t, t) \rightarrow 1$ as $t \rightarrow \infty$ for all $\tilde{z} \geq 0$, $c \geq 0$

(b) $g(x)=O\left(e^{-\sigma x} x^{-n}\right)$ as $x \rightarrow \infty$ with $\sigma>0$ and $-\infty<n<\infty$, a permanent form travelling wave evolves with propagation speed $v(t) \sim v_{0} \sqrt{D}+v_{1} \sqrt{D} t^{-1}$ as $t \rightarrow \infty$, where

(i) $v_{0}=\sigma \sqrt{D}+1 / \sigma \sqrt{D}, v_{1}=-n / \sigma \sqrt{D}$ for $0<\sigma<1 / \sqrt{D},-\infty<n<\infty$,

(ii) $v_{0}=2, v_{1}=-\frac{1}{2}(n+1)$ for $\sigma=1 / \sqrt{D}, n \leq 2$,

(iii) $v_{0}=2, v_{1}=-3 / 2$ for $\sigma=1 / \sqrt{D}, n \geq 2$ or $\sigma>1 / \sqrt{D},-\infty<n<\infty$, in the sense that

$$
\begin{aligned}
& {\left[\alpha\left(\tilde{z}+c_{0} \sqrt{D} t+c_{1} \sqrt{D} \log t, t\right), \beta\left(\tilde{z}+c_{0} \sqrt{D} t+c_{1} \sqrt{D} \log t, t\right)\right]} \\
& \quad \rightarrow \begin{cases}{[0,1]} & \text { for both } c_{0}<v_{0} \text { and } c_{0}=v_{0} \text { with } c_{1}<v_{1}, \\
{\left[\alpha^{*}(\tilde{z}), \beta^{*}(\tilde{z})\right]} & \text { for } c_{0}=v_{0}, c_{1}=v_{1}, \\
{[1,0]} & \text { for both } c_{0}>v_{0} \text { and } c_{0}=v_{0} \text { with } c_{1}>v_{1},\end{cases}
\end{aligned}
$$

as $t \rightarrow \infty$ with $\tilde{z}$ fixed, where $\alpha^{*}(\tilde{z}), \beta^{*}(\tilde{z})$ is the permanent form travelling wave solution of equations (6) with propagation speed $v_{0} \sqrt{D}$;

(c) $g(x)$ has compact support, a permanent form travelling wave evolves with propagation speed $v(t) \sim 2 \sqrt{D}-\frac{3}{2} \sqrt{D} t^{-1}$ as $t \rightarrow \infty$, in the sense defined above.

Clearly Result 1.1 encompasses all of the results that are suggested by the earlier work on the initial value problem (9). In addition, Result 1.1 shows that travelling wave solutions of the initial value problem (6), (7) can propagate at any speed $v(t) \geq$ $v_{\min }(t)$, where $v_{\min } \sim 2 \sqrt{D}-\frac{3}{2} \sqrt{D} t^{-1}$ as $t \rightarrow \infty$, and that $v(t)$ is dependent upon $\sigma$ and $n$ when $g(x)=O\left(e^{-\sigma x} x^{-n}\right)$ as $x \rightarrow \infty$. 
In Sec. 2 we analyse the asymptotic solution of the initial value problem (6), (7) for $t \gg 1$ with $D>0$ and various classes of initial input function $g(x)$. This allows us to formally establish Result 1.1. In Sec. 3 that obtain numerical solutions of the initial value problem (6), (7) that are consistent with Result 1.1.

2. Asymptotic solution of the initial value problem (6), (7) for $t \gg 1$. In this section we construct the asymptotic solution of the initial value problem (6), (7) for $t \gg 1$ when $g(x)$ is in one of the three classes of functions, $G_{1}, G_{2}$, or $G_{3}$ defined by

$$
\begin{aligned}
& G_{1}=\left\{g(x): g(x) \sim k x^{-n} \text { as } x \rightarrow \infty, \text { for some } k>0, n>1\right\}, \\
& G_{2}=\left\{g(x): g(x) \sim k e^{-\sigma x} x^{-n} \text { as } x \rightarrow \infty,\right. \\
& \quad \text { for some } k>0, \sigma>0,-\infty<n<\infty\}, \\
& G_{3}=\{g(x): g(x) \equiv 0 \text { for } x>\lambda, \text { for some } \lambda>0\} .
\end{aligned}
$$

2.1. $g(x) \in G_{1}$. First: we determine the asymptotic solution of the initial value problem (6), (7) for $x \gg 1, t=O(1)$. We can then determine the domain of validity of this solution as $t \rightarrow \infty$. Finally, we complete the asymptotic solution for $t \gg 1$ by introducing three further asymptotic regions.

The initial conditions (7a) show that

$$
\alpha \rightarrow 1, \quad \beta \sim k_{0} x^{-n} \quad \text { as } x \rightarrow \infty, \text { when } t=0,
$$

where $k_{0}=k \beta_{0}$, which suggests that we should seek a solution of Eq. (6) in the form

$$
\alpha=1+o(1), \quad \beta=x^{-n} \hat{\beta}(t)+o\left(x^{-n}\right) \quad \text { as } x x \rightarrow \infty, \text { with } t=O(1) .
$$

In terms of the scaled variables (12a), Eq. (6b) becomes, at leading order in $x$,

$$
\hat{\beta}_{t}=\hat{\beta} \text {, }
$$

and the solution of Eq. (12b), which satisfies the initial condition $\hat{\beta}=k_{0}$ when $t=0$, is

$$
\hat{\beta}=k_{0} e^{t} .
$$

In terms of the original variables we therefore have

$$
\alpha=1+o(1), \quad \beta=k_{0} e^{t} x^{-n}+o\left(x^{n}\right) \quad \text { as } x \rightarrow \infty \text { with } t=O(1) .
$$

As $t \rightarrow \infty$, this approximation remains valid provided $e^{t} x^{-n} \ll 1$, and hence for $x \gg e^{t / n}$, which we label as region A. In order to develop the asymptotic solution when $x=O\left(e^{t / n}\right)$ as $t \rightarrow \infty$, we introduce region I where from expansions (13) appropriate scaled variables are

$$
\tilde{x}=x e^{-t / n}, \quad \alpha=O(1), \quad \beta=O(1) \quad \text { as } t \rightarrow \infty, \text { with } \tilde{x}=O(1) .
$$

In terms of the scaled variables $(14 a)$, Eq. $(6 a, b)$ become, at leading order,

$$
\tilde{x} \alpha_{x}=n \alpha \beta, \quad \tilde{x} \beta_{\tilde{x}}=-n \alpha \beta,
$$

which are to be solved subject to the matching condition with region $\mathrm{A}$,

$$
\alpha \rightarrow 1, \quad \beta \sim k_{0} \tilde{x}^{-n} \quad \text { as } \tilde{x} \rightarrow \infty .
$$


This boundary value problem is readily solved to give the solution

$$
\alpha=\frac{\tilde{x}^{n}}{k_{0}+\tilde{x}^{n}}+o(1), \quad \beta=\frac{k_{0}}{k_{0}+\tilde{x}^{n}}+o(1) \quad \text { as } t \rightarrow \infty \text { with } \tilde{x}=O(1) .
$$

Although these approximations satisfy the boundary conditions (7b) at $x=0$, expression (8), which is valid when $D=1$, shows that $\alpha+\beta=1+O\left(t^{-1 / 2}\right)$ as $t \rightarrow \infty$ for $x=O(1)$. The nature of Eq. (6) suggests that this is also the case for all $D>0$, and therefore, we must introduce further asymptotic regions in order to complete the solution. Expansions (15) suggest that scaled variables for the first of these, region IIa, are

$$
\alpha=o\left(t^{-m}\right) \quad \forall m>0, \quad \beta=1+(D t)^{-1 / 2} \hat{\beta} \quad \text { as } t \rightarrow \infty \text { with } x=O(1) .
$$

If we take $\alpha$ to be algebraically small as $t \rightarrow \infty$ in region IIa, we find that the solution obtained at leading order will not match with the solution in region I as $x \rightarrow \infty$. However, the behaviour of the exponentially small concentration $\alpha$ in region IIa is not pursued further in the present paper. In terms of the scaled variables (16a), Eq. (6b) becomes

$$
\hat{\beta}_{t}=D \hat{\beta}_{x x}+\frac{1}{2} t^{-1} \hat{\beta}
$$

up to terms exponentially small as $t \rightarrow \infty$, and we expand $\hat{\beta}$ as

$$
\hat{\beta}=\hat{\beta}_{0}(x)+(D t)^{-1} \hat{\beta}_{1}(x)+o\left((D t)^{-1}\right) \quad \text { as } t \rightarrow \infty \text { with } x=O(1) .
$$

After substituting expansion (16c) into equation (16b) and solving for $\hat{\beta}_{0}$ and $\hat{\beta}_{1}$, we obtain the solutions

$$
\hat{\beta}_{0}=\hat{b}_{0}, \quad \hat{\beta}_{1}=-\frac{1}{4} \hat{b}_{0} x^{2}+\hat{b}_{1},
$$

which satisfy the boundary conditions $(7 \mathrm{~b})$ at $x=0$. The constants $\hat{b}_{0}$ and $\hat{b}_{1}$ are dependent upon the details of the initial input function $\beta_{0} g(x)$ and cannot be determined in this large time asymptotic solution. Expansion (16c) now becomes

$$
\hat{\beta}=\hat{b}_{0}+(D t)^{-1}\left(-\frac{1}{4} \hat{b}_{0} x^{2}+\hat{b}_{1}\right)+o\left((D t)^{-1}\right) \quad \text { as } t \rightarrow \infty \text { with } x=O(1) .
$$

We next observe that this expansion becomes nonuniform as $x \rightarrow \infty$, in particular, when $x=O\left(t^{1 / 2}\right)$. We must therefore introduce a further asymptotic region, region IIb, where, from expansion (17b), appropriate scaled variables are

$$
\begin{aligned}
\bar{x}=x(D t)^{-1 / 2}, \quad \alpha=o\left(t^{-m}\right) \forall m>0, \quad \beta=1+(D t)^{-1 / 2} \bar{\beta} \\
\text { as } t \rightarrow \infty \text { with } \bar{x}=O(1) .
\end{aligned}
$$

In terms of these scaled variables, Eq. $(6 \mathrm{~b})$ becomes

$$
t \bar{\beta}_{t}=\bar{\beta}_{\overline{x x}}+\frac{1}{2} \bar{x} \bar{\beta}_{\bar{x}}+\frac{1}{2} \bar{\beta}
$$

and we expand $\bar{\beta}$ as

$$
\bar{\beta}=\bar{\beta}_{0}(\bar{x})+(D t)^{-1} \bar{\beta}_{1}(\bar{x})+o\left((D t)^{-1}\right) \quad \text { as } t \rightarrow \infty \text { with } \bar{x}=O(1) .
$$


After substituting expansion (18c) into Eq. (18b) and solving the resulting equations for $\bar{\beta}_{0}$ and $\bar{\beta}_{1}$, we obtain the solutions

$$
\bar{\beta}_{0}=\hat{b}_{0} e^{-\bar{x}^{2} / 4}, \quad \bar{\beta}_{1}=\hat{b}_{1}\left(1-\frac{1}{2} \bar{x}^{2}\right) e^{-\bar{x}^{2} / 4},
$$

which match with the expansion (17b) in region IIa as $\bar{x} \rightarrow 0$. Expansion (18c) is now determined as

$$
\begin{array}{r}
\bar{\beta}=\hat{b}_{0} e^{-\bar{x}^{2} / 4}+(D t)^{-1} \hat{b}_{1}\left(1-\frac{1}{2} \bar{x}^{2}\right) e^{-\bar{x}^{2} / 4}+o\left((D t)^{-1}\right) \\
\text { as } t \rightarrow \infty \text { with } \bar{x}=O(1) .
\end{array}
$$

This expansion develops a nonuniformity as $\bar{x} \rightarrow \infty$, when $\bar{\beta}$ becomes exponentially small. This then enables expansion (19b) as $\bar{x} \rightarrow \infty$ to match with expansion (15) in region $\mathrm{I}$ as $\bar{x} \rightarrow 0$ to all algebraic orders in $t^{-1}$. This completes the solution of the initial value problem (6), (7) for $t \gg 1$ when $g(x) \in G_{1}$. We can summarize the leading order solution in each region:

$$
\begin{aligned}
& \text { Region IIa. } x=O(1), \quad \alpha=o\left(t^{-m}\right) \quad \forall m>0 \text {, } \\
& \beta=1+\hat{b}_{0}(D t)^{-1 / 2}+O\left((D t)^{-1}\right) \quad \text { as } t \rightarrow \infty ; \\
& \text { REGION IIb. } x=O\left(t^{1 / 2}\right), \quad \alpha=o\left(t^{-m}\right) \quad \forall m>0 \text {, } \\
& \beta=1+\hat{b}_{0} e^{-\bar{x}^{2} / 4}(D t)^{-1 / 2}+O\left((D t)^{-1}\right) \\
& \text { as } \left.t \rightarrow \infty \text {, where } \bar{x}=x /(D t)^{1 / 2}\right\} \\
& \text { REGION I. } \quad x=O\left(e^{t / n}\right), \quad \alpha=\frac{\tilde{x}^{n}}{k_{0}+\tilde{x}^{n}}+o(1), \\
& \beta=\frac{k_{0}}{k_{0}+\tilde{x}^{n}}+o(1) \quad \text { as } t \rightarrow \infty \text {, where } \tilde{x}=x e^{-t / n} .
\end{aligned}
$$

Region $\mathrm{A}$ is passive and simply transmits the structure in region $\mathrm{I}$, which remains uniform as $\tilde{x} \rightarrow \infty$. Therefore, we can analyse the asymptotic solution in terms of the regions I, IIa, and IIb alone. In regions IIa and IIb, $\alpha$ is exponentially small and $\beta \rightarrow 1$ as $t \rightarrow \infty$ via algebraically small terms. In terms of the chemical reaction, the reactant, $A$, is effectively completely consumed, whilst the concentration of the autocatalyst, $B$, diffuses down to unity. In region I, the concentrations $\alpha$ and $\beta$ adjust to their far-field values of unity and zero, respectively, over a length scale of $O\left(e^{t / n}\right)$ as $t \rightarrow \infty$. The solution in this region represents a phase wave that is driven by reaction alone with negligible diffusion. The numerical solution of the initial value problem (6), (7) is illustrated in Fig. 1(a) (see p. 350) in the case $D=1, n=4$ at $t=20$, and clearly displays the features described by (20) above. Numerical solutions of the initial value problem (6), (7) are discussed in detail in Sec. 3.

Finally, we note that we can construct a similar asymptotic solution when $g(x)$ is such that $e^{\sigma x} g(x) \rightarrow \infty$ as $x \rightarrow \infty$ for all $\sigma>0$, with

$$
\begin{array}{r}
\alpha=\frac{1-\beta_{0} g(x)}{1+\beta_{0} g(x)\left(e^{t}-1\right)}+o(1), \quad \beta=\frac{\beta_{0} g(x) e^{t}}{1+\beta_{0} g(x)\left(e^{t}-1\right)}+o(1) \\
\text { as } t \rightarrow \infty \text { for } g(x) e^{t}=O(1)
\end{array}
$$




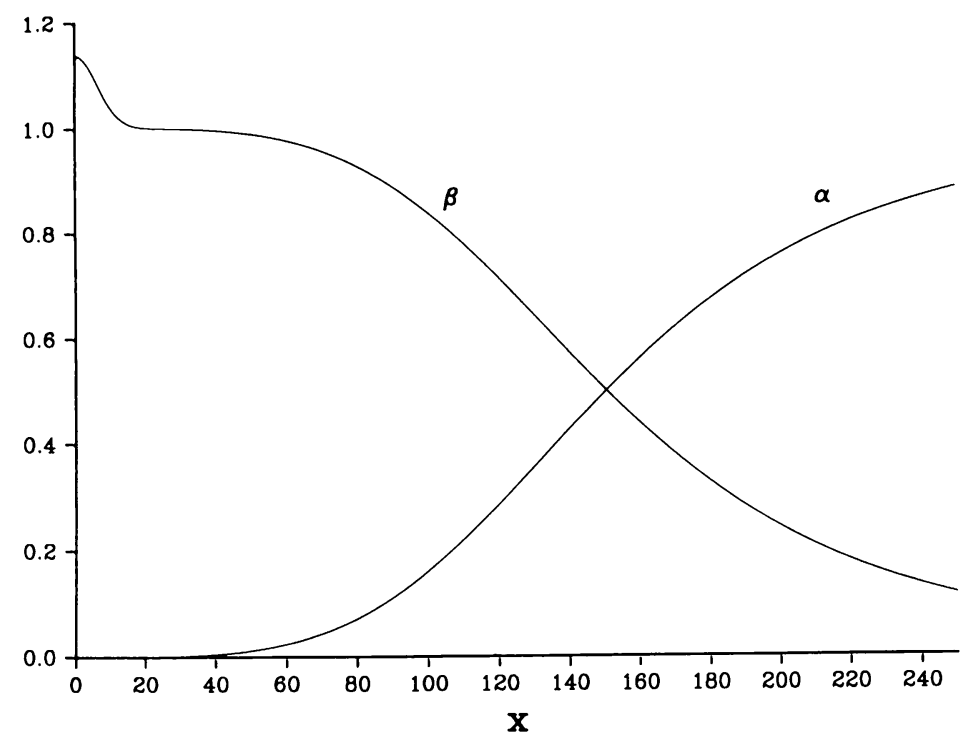

Fig. 1(a). A graph of the solution of the initial value problem (6), (7) when $D=1$ and $g(x)$ is given by Eq. (61) (on p. 369) with $n=4$, at $t=20$.

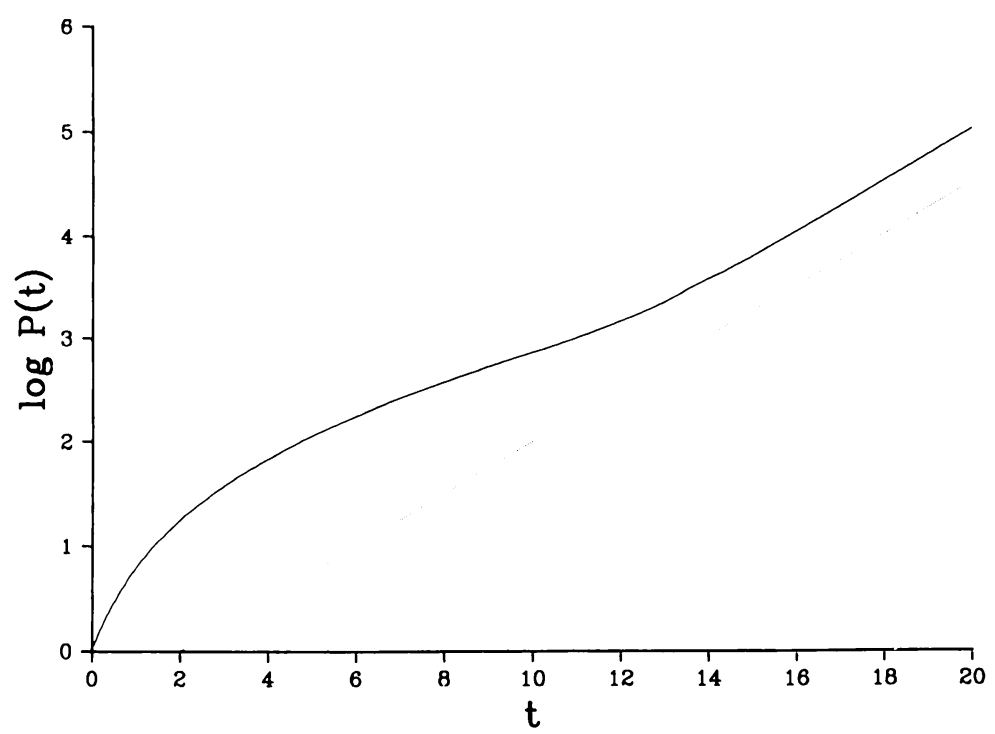

Fig. 1(b). A graph of the function $\log P(t)$. The broken line has gradient $1 / n=\frac{1}{4}$ in this case.

in region $\mathrm{I}$, with regions IIa, $\mathrm{b}$ unchanged. This solution again represents a phase wave. When $e^{\sigma x} g(x)=O(1)$ as $x \rightarrow \infty$ for some $\sigma>0$ the solution (21) is no longer valid. In this case the diffusion terms in Eq. (6a, b) appear in the leading order equation in region $\mathrm{A}$ and the solution has a completely different structure, which we discuss in detail below. 
2.2. $g(x) \in G_{2} \cup G_{3}$. As before, we begin by determining the asymptotic solution of the initial value problem (6), (7) for $x \gg 1$ with $g(x) \in G_{2}$ and also for $g(x) \in$ $G_{3}$. We find that these two solutions have similar domains of validity as $t \rightarrow \infty$, and we complete the asymptotic solution for $t \gg 1$ by introducing further asymptotic regions which have a similar structure for both $g(x) \in G_{2}$ and $g(x) \in G_{3}$. When $g(x) \in G_{2}$ there are three cases to consider, which correspond to (b)(i), (ii), and (iii) of Result 1.1.

(a) $g(x) \in G_{2}$. The initial conditions (7a) show that

$$
\alpha \rightarrow 1, \quad \beta \sim k_{0} e^{-\sigma x} x^{-n} \quad \text { as } x \rightarrow \infty, \text { when } t=0,
$$

where $k_{0}=k \beta_{0}$, which suggests that we should seek a solution of Eq. (6) of the form

$$
\alpha=1+o(1), \quad \beta=e^{-x \phi(x, t)} \quad \text { as } x \rightarrow \infty \text { with } t=O(1) .
$$

Here, the function $\phi(x, t)$ is positive and of $O(1)$ as $x \rightarrow \infty$. In terms of the variables $(22 \mathrm{~b})$, Eq. (6b) becomes

$$
\phi_{t}=x^{-1}\left\{D\left(2 \phi_{x}+x \phi_{x x}-\phi^{2}-2 x \phi \phi_{x}-x^{2} \phi_{x}^{2}\right)-1\right\},
$$

and we expand $\phi$ as

$$
\phi=\phi_{0}+x^{-1} \log x \phi_{1}+x^{-1} \phi_{2}+o\left(x^{-1}\right) \quad \text { as } x \rightarrow \infty \text { with } t=O(1) \text {. }
$$

After substituting Eq. (22d) into Eq. (22c) and solving for $\phi_{0}, \phi_{1}$, and $\phi_{2}$, we obtain the solutions

$$
\phi_{0}=\sigma, \quad \phi_{1}=n, \quad \phi_{2}=-\left(D \sigma^{2}+1\right) t-\log k_{0},
$$

which satisfy the initial conditions given by (22a). Expansion (22d) now gives

$$
\begin{aligned}
\phi=\sigma+n x^{-1} \log x+x^{-1}\left(-\left(D \sigma^{2}+1\right) t-\log k_{0}\right)+o\left(x^{-1}\right) \\
\text { as } x \rightarrow \infty \text { with } t=O(1),
\end{aligned}
$$

and in terms of the original variables,

$$
\beta \sim k_{0} x^{-n} \exp [-\sigma\{x-(D \sigma+1 / \sigma) t\} \quad \text { as } x \rightarrow \infty \text { with } t=O(1) .
$$

This solution suggests that a travelling wave evolves with propagation speed $D \sigma+$ $1 / \sigma$, and we show below that this interpretation is correct, but only for $0<\sigma<$ $1 / \sqrt{D}$. For $\sigma \geq 1 / \sqrt{D}$, the structure of the asymptotic solution is more complicated, and we find that a travelling wave evolves with propagation speed $2 \sqrt{D}$. As $t \rightarrow \infty$, expansion (23b) remains uniform for $x \gg t$, which we label as region $\mathrm{A}$, and in order to develop the asymptotic solution when $x=O(t)$ as $t \rightarrow \infty$, we introduce region $\mathrm{I}$, where $x=O(t)$. Before we continue in developing the asymptotic solution in region I for $t \gg 1$, we consider the case when $g(x) \in G_{3}$.

(b) $g(x) \in G_{3}$. The initial conditions (7a) show that

$$
\alpha \rightarrow 1, \quad \beta \rightarrow 0 \quad \text { as } t \rightarrow 0 \text { for } x \gg 1 .
$$

Since Eq. (6b) is simply a diffusion equation with a source term, this suggests that we should seek a solution of the form

$$
\alpha=1+o(1), \quad \beta=e^{-x^{2} \psi(x, t)} \quad \text { as } x \rightarrow \infty \text { with } t=O(1),
$$


where the function $\psi(x, t)$ is positive as $x \rightarrow \infty$. In terms of the variables (25a) Eq. (6b) becomes

$$
\psi_{t}=D\left(\psi_{x x}+4 x^{-1} \psi_{x}+2 x^{-2} \psi-4 \psi^{2}-4 x \psi \psi_{x}-x^{2} \psi_{x}^{2}\right)-x^{-2},
$$

and we expand $\psi$ as

$$
\psi=\psi_{0}+x^{-1} \psi_{1}+x^{-2} \psi_{2}+o\left(x^{-2}\right) \quad \text { as } x \rightarrow \infty \text { with } t=O(1) .
$$

After substituting expansion (25c) into Eq. (25b) and solving for $\psi_{0}, \psi_{1}$, and $\psi_{2}$, we obtain the solutions

$$
\psi_{0}=\frac{1}{4 D t}, \quad \psi_{1}=\frac{K_{1}}{2 D t}, \quad \psi_{2}=-t+\frac{1}{2} \log t+\frac{k_{1}^{2}}{4 D t}-\log k_{2},
$$

which satisfy the initial conditions given by (24), where $k_{1}$ and $k_{2}$ are constants to be determined. Expansion (25c) now becomes

$$
\begin{aligned}
\psi=\frac{1}{4 D t}+\frac{k_{1} x^{-1}}{2 D t}+x^{-2}\left(-t+\frac{1}{2} \log t+\frac{k_{1}^{2}}{4 D t}-\log k_{2}\right) & +o\left(x^{-2}\right) \\
\text { as } x & \rightarrow \infty \text { with } t=O(1),
\end{aligned}
$$

and in terms of the original variables,

$$
\beta \sim k_{2} \exp \left\{-\frac{\left(x+k_{1}\right)^{2}}{4 D t}+t-\frac{1}{2} \log t\right\} \quad \text { as } x \rightarrow \infty \text { with } t=O(1) .
$$

This solution suggests that a travelling wave evolves with propagation speed $v(t)=$ $2 \sqrt{D}+O\left(t^{-1}\right)$ as $t \rightarrow \infty$, a result confirmed in the analysis below. As $t \rightarrow \infty$, expansion (26b) remains uniform for $x \gg t$, which we again label as region $\mathrm{A}$, and we must introduce region I, where $x=O(t)$, in order to continue the solution.

We now develop the asymptotic solution in region I as $t \rightarrow \infty$ for both of the cases (a) and (b). In the following analysis we find that there are four different types of asymptotic structures that the solution can display, corresponding to the cases $(b)(i)$, (ii), (iii) and (c) of Result 1.1.

We begin by noting that there is no nontrivial asymptotic solution of Eq. (6) in terms of the variables $X=x t^{-1}$ and $t$ as $t \rightarrow \infty$, which will match as $X \rightarrow \infty$ to the solution in region A with $x=O(t)$. This leads us to define scaled variables for region $I$ as

$$
y=x-\sqrt{D} \gamma(t), \quad \alpha=O(1), \quad \beta=O(1), \quad \begin{aligned}
\gamma & =O(t) \\
\text { as } t & \rightarrow \infty \text { with } y=O(1) .
\end{aligned}
$$

The dependence of the solution upon the travelling coordinate $y$ is also suggested by the form of the solution in region A given by $(23 \mathrm{c})$ or $(26 \mathrm{c})$. In terms of the variables (27a), Eqs. (6) become

$$
\begin{gathered}
\frac{\partial^{2} \alpha}{\partial y^{2}}+\sqrt{D} \frac{d \gamma}{d t} \frac{\partial \alpha}{\partial y}-\alpha \beta=\frac{\partial \alpha}{\partial t}, \\
D \frac{\partial^{2} \beta}{\partial y^{2}}+\sqrt{D} \frac{d \gamma}{d t} \frac{\partial \beta}{\partial y}+\alpha \beta=\frac{\partial \beta}{\partial t},
\end{gathered}
$$


which are to be solved subject to matching with the solution in region A as $y \rightarrow \infty$.

We expand $\alpha, \beta$, and $\gamma$ as

$$
\begin{gathered}
\alpha=\alpha_{0}+t^{-1 / 2} \alpha_{1}+t^{-1} \alpha_{2}+o\left(t^{-1}\right), \quad \beta=\beta_{0}+t^{-1 / 2} \beta_{1}+t^{-1} \beta_{2}+o\left(t^{-1}\right) \\
\text { as } t \rightarrow \infty \text { with } y=O(1), \\
\gamma=v_{0} t+v_{1} \log t+o(\log t) \quad \text { as } t \rightarrow \infty,
\end{gathered}
$$

and note that it will become apparent that expansions of this form are necessary in order to construct a consistent asymptotic solution that matches the solution in region A. In particular, an expansion for $\gamma$, which includes terms of $O\left(t^{p}\right)$ for $0<p<1$, generates terms in the expansions of $\alpha$ and $\beta$ that do not satisfy the appropriate matching conditions as $y \rightarrow \infty$. On substituting expansions (28a, b) into equations $(27 \mathrm{~b}, \mathrm{c})$ we obtain the leading order equations

$$
\alpha_{0}^{\prime \prime}+v_{0} \sqrt{D} \alpha_{0}^{\prime}-\alpha_{0} \beta_{0}=0, \quad D \beta_{0}^{\prime \prime}+v_{0} \sqrt{D} \beta_{0}^{\prime}+\alpha_{0} \beta_{0}=0,
$$

which are to be solved subject to the matching condition

$$
\alpha_{0} \rightarrow 1, \beta_{0} \rightarrow 0 \text { as } y \rightarrow \infty ;
$$

we also require

$$
\alpha_{0}>0, \beta_{0}>0 \text { for }-\infty<y<\infty .
$$

The nonlinear eigenvalue problem $(29 a, b, c)$ was studied in [2] where we showed that for $0 \leq v_{0}<2$ all nontrivial solutions of the boundary value problem (29a, b) become negative for some finite value of $y$, and therefore do not satisfy (29c). However, for each $v_{0} \geq 2$ a travelling wave solution exists, which is unique up to translation, satisfies $(29 c)$, and has

$$
\alpha_{0} \sim a_{0} e^{\mu_{+} y}, \quad \beta_{0} \sim 1-\frac{a_{0} e^{\mu_{+} y}}{D \mu_{+}^{2}+v_{0} \sqrt{D} \mu_{+}} \quad \text { as } y \rightarrow-\infty,
$$

where $\mu_{+}=-\frac{1}{2}\left\{v_{0} \sqrt{D}-\sqrt{D v_{0}^{2}+4}\right\}$ and

$$
\beta_{0} \sim \begin{cases}\alpha_{0} \rightarrow 1, & \\ \left(A_{0}\left(a_{0}\right) y+B_{0}\left(a_{0}\right)\right) e^{-y / \sqrt{D}} & v_{0}=2, \\ C_{0}\left(a_{0}\right) e^{\lambda_{+} y} & v_{0}>2, \text { as } y \rightarrow \infty,\end{cases}
$$

where $\lambda_{+}=\left\{-v_{0}+\sqrt{v_{0}^{2}-4}\right\} / 2 \sqrt{D}$, with the translational constant $a_{0}$ to be determined, $A_{0}>0$ and $C_{0}>0$. This completes the leading order term in region I. Before proceeding to higher order terms in region I we note that, as was the case for $g(x) \in G_{1}$, we expect, via (8), that the expansions (28) become nonuniform as $y \rightarrow-\infty$. We examine this next.

As before, we introduce region IIa where expansion (30a) suggests that appropriate scaled variables are

$$
\alpha=t^{-v_{1} \sqrt{D} \mu_{+}} e^{-v_{0} \sqrt{D} \mu_{+} t} \hat{\alpha}, \quad \beta=1+(D t)^{-1 / 2} \hat{\beta} \quad \text { as } t \rightarrow \infty \text { for } x=O(1) .
$$


In this case, we can readily obtain the leading order behaviour of $\alpha$, and we expand $\hat{\alpha}$ as

$$
\hat{\alpha}=\hat{\alpha}_{0}(x)+o(1) \quad \text { as } t \rightarrow \infty \text { with } x=O(1),
$$

whilst the expansion for $\hat{\beta}$ is again given by (17b). After rewriting Eq. (6a) in terms of the scaled variables (31a) and substituting expansion (31b) for $\hat{\alpha}$, we obtain

$$
\hat{\alpha}_{0}^{\prime \prime}-\mu_{+}^{2} \hat{\alpha}_{0}=0 \text {, }
$$

which is to be solved subject to the boundary condition,

$$
\hat{\alpha}_{0}^{\prime}(0)=0 \text {. }
$$

We can readily integrate Eq. (32a) to obtain

$$
\hat{\alpha}_{0}=\hat{a}_{0} \cosh \mu_{+} x \text {, }
$$

which satisfies the boundary condition $(32 \mathrm{~b})$, and $\hat{a}_{0}$ is a positive constant to be determined. In region IIb, appropriate scaled variables are, from (17b) and (32c),

$$
\begin{aligned}
& \bar{x}=x(D t)^{-1 / 2}, \quad \alpha=t^{-v_{1} \sqrt{D} \mu_{+}} e^{-v_{0} \sqrt{D} \mu_{+} t} e^{t^{1 / 2} \bar{A}}, \quad \beta=1+(D t)^{-1 / 2} \bar{\beta} \\
& \text { as } t \rightarrow \infty \text { with } \bar{x}=O(1) \text {. }
\end{aligned}
$$

We expand $\bar{A}$ as

$$
\bar{A}=\bar{A}_{0}+t^{-1 / 2} \bar{A}_{1}+o\left(t^{-1 / 2}\right) \quad \text { as } t \rightarrow \infty \text { with } \bar{x}=O(1),
$$

and the expansion for $\bar{\beta}$ is again given by (19b). After rewriting Eq. (6a) in terms of the scaled variables (33a) and substituting expansion (33b) for $\bar{A}$, we obtain the equations

$$
\left(\bar{A}_{0}^{\prime}\right)^{2}=D \mu_{+}^{2}, \quad \frac{1}{2}\left(\bar{A}_{1}-\bar{x} \bar{A}_{1}^{\prime}\right)=2 D^{-1} \bar{A}_{0}^{\prime} \bar{A}_{1}^{\prime}+D^{-1} \bar{A}_{0}^{\prime \prime}-D^{-1 / 2} \bar{\beta}_{0},
$$

which are to be solved subject to the matching conditions

$$
\bar{A}_{0} \sim \mu_{+} \sqrt{D} \bar{x}, \quad \bar{A}_{1} \sim \log \hat{a}_{0} \quad \text { as } \bar{x} \rightarrow 0
$$

with the solution in region IIa, and

$$
\bar{A}_{0} \sim \mu_{+} \sqrt{D} \bar{x}, \quad \bar{A}_{1} \sim \log a_{0} \quad \text { as } \bar{x} \rightarrow \infty
$$

with the solution in region I. We can readily solve these two boundary value problems to obtain the solutions

$$
\bar{A}_{0}=\mu_{+} \sqrt{D} \bar{x}, \quad \bar{A}_{1}=\log a_{0}-\frac{\hat{b}_{0} \sqrt{\pi D}}{2 \mu_{+}} \operatorname{erfc}\left(\frac{1}{2} \bar{x}\right),
$$

and find that the constant $\hat{a}_{0}$ satisfies

$$
\log \hat{a}_{0}=\log a_{0}-\frac{\hat{b}_{0} \sqrt{\pi D}}{2 \mu_{+}} .
$$

This completes the asymptotic solution in regions IIa and IIb and we return to the solution at higher order in region $\mathrm{I}$.

At $O\left(t^{-1 / 2}\right)$ Eqs. $(27 \mathrm{~b}, \mathrm{c})$ become 


$$
\begin{gathered}
\alpha_{1}^{\prime \prime}+v_{0} \sqrt{D} \alpha_{1}^{\prime}-\beta_{0} \alpha_{1}-\alpha_{0} \beta_{1}=0, \\
D \beta_{1}^{\prime \prime}+v_{0} \sqrt{D} \beta_{1}^{\prime}+\beta_{0} \alpha_{1}+\alpha_{0} \beta_{1}=0,
\end{gathered}
$$

and are to be solved subject to the matching conditions with regions A and IIb,

$$
\alpha_{1} \rightarrow 0, \quad \beta_{1} \rightarrow 0 \quad \text { as }|y| \rightarrow \infty .
$$

The boundary value problem $(35 \mathrm{a}, \mathrm{b}, \mathrm{c})$ is linear and homogeneous, and it is readily verified that

$$
\alpha_{1}=b_{1} \alpha_{0}^{\prime}, \quad \beta_{1}=b_{1} \beta_{0}^{\prime}
$$

provides a solution for any constant $b_{1}>0$. However, an examination of the local behaviour of solutions of equations (35a, b) as $y \rightarrow-\infty$ also shows that (35d) is the only solution to this boundary value problem. We show below that we require $b_{1}$ to be nonzero in order to satisfy a later matching condition, and note that the inclusion of the terms of $O\left(t^{-1 / 2}\right)$ in expansions (28a) corrects a small inaccuracy in Merkin and Needham [12].

At $O\left(t^{-1}\right)$ Eqs. $(27 \mathrm{~b}, \mathrm{c})$ become

$$
\begin{gathered}
\alpha_{2}^{\prime \prime}+v_{0} \sqrt{D} \alpha_{2}^{\prime}-\beta_{0} \alpha_{2}-\beta_{1} \alpha_{1}-\alpha_{0} \beta_{2}=v_{0} \alpha_{0}^{\prime}, \\
D \beta_{2}^{\prime \prime}+v_{0} \sqrt{D} \beta_{2}^{\prime}+\beta_{0} \alpha_{2}+\beta_{1} \alpha_{1}+\alpha_{0} \beta_{2}=-v_{0} \beta_{0}^{\prime}
\end{gathered}
$$

and are to be solved subject to the matching conditions

$$
\alpha_{2} \rightarrow 0, \beta_{2} \rightarrow 0 \quad \text { as }|y| \rightarrow \infty .
$$

When $D=1$ we can readily obtain the solution of this boundary value problem as

$$
\beta_{2}=-\alpha_{2}=b_{2} \alpha_{0}^{\prime}-b_{1} \alpha_{0}^{\prime} \int_{0}^{y} \frac{e^{-v_{0} u}}{\alpha_{0}^{\prime 2}(u)} \int_{0}^{u} e^{v_{0} s}\left\{\frac{v_{1}}{b_{1}} \alpha_{0}^{\prime 2}(s)+b_{1} \alpha_{0}^{\prime 3}(s)\right\} d s d u .
$$

No solvability condition arises and the solution exists for each $-\infty<v_{1}<\infty$. When $D \neq 1$ the boundary value problem (36) cannot be readily integrated. However, we can show that no solvability condition arises and obtain the asymptotic behaviour of $\alpha_{2}$ and $\beta_{2}$ for $y \gg 1$ from Eqs. (36a, b) as

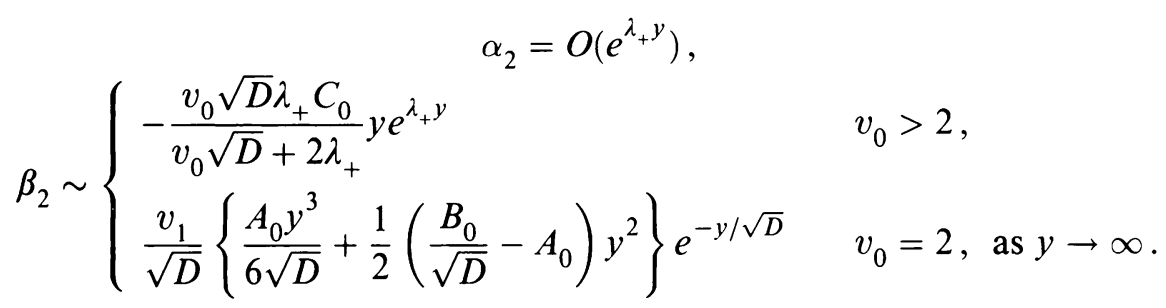

The detailed behaviour of $\alpha_{2}$ as $y \rightarrow \infty$ is dependent upon $v_{0}$ and $D$ and is not required for the purposes of this paper. Expansions (28a) now show that

$$
\alpha \sim 1+O\left(e^{\lambda_{+} y}\right),
$$




$$
\beta \sim\left\{\begin{array}{c}
C_{0} e^{\lambda_{+} y}+t^{-1 / 2} b_{1} \lambda_{+} C_{0} e^{\lambda_{+} y}-t^{-1}\left(\frac{v_{0} \sqrt{D} \lambda_{+} C_{0}}{v_{0} \sqrt{D}+2 \lambda_{+}}\right) y e^{\lambda_{+} y}+o\left(t^{-1}\right) \quad v_{0}>2, \\
\left(A_{0} y+B_{0}\right) e^{-y / \sqrt{D}}+t^{-1 / 2} b_{1}\left\{-\frac{A_{0} y}{\sqrt{D}}+A_{0}-\frac{B_{0}}{\sqrt{D}}\right\} e^{-y / \sqrt{D}} \\
+t^{-1} \frac{v_{1}}{\sqrt{D}}\left\{\frac{A_{0} y^{3}}{6 \sqrt{D}}+\frac{1}{2}\left(\frac{B_{0}}{\sqrt{D}}-A_{0}\right) y^{2}\right\} e^{-y / \sqrt{D}}+o\left(t^{-1}\right) \\
v_{0}=2, \text { as } t \rightarrow \infty \text { for } y \gg 1 .
\end{array}\right.
$$

We now direct our attention to the behaviour of the concentration $\beta$ alone, since this allows us to determine $v_{0}$ and $v_{1}$. As expected, expansions (38) become nonuniform as $y \rightarrow \infty$, in particular, when $y=O(t)$ for $v_{0}>2$ and when $y=O\left(t^{1 / 2}\right)$ for $v_{0}=2$, and therefore, further regions are required between region $I$ and the final region A where $y \gg t$. We examine these two cases separately.

2.2.1. $v_{0}>2$. In order to develop the asymptotic solution of the initial value problem (6), (7) when $y=O(t)$ as $t \rightarrow \infty$, we introduce region III where appropriate scaled variables are, from expansions (38),

$$
\begin{aligned}
Y=y / D^{1 / 2} t, \quad \alpha=1+o\left(t^{-m}\right) \quad \forall m>0, \quad \beta=e^{\lambda_{+} D^{1 / 2} t Y} B \\
\text { as } t \rightarrow \infty \text { with } Y=O(1) \text { and } Y>0 .
\end{aligned}
$$

In terms of the scaled variables (39a), Eq. (6b) becomes, at leading order,

$$
\left\{Y+\sqrt{v_{0}^{2}-4}\right\} B_{Y}+\lambda_{+} v_{1} \sqrt{D} B=0
$$

which is to be solved subject to matching with expansion (38) in region I, which leads to

$$
B \rightarrow C_{0} \quad \text { as } Y \rightarrow 0
$$

The appropriate solution is readily obtained as

$$
B=C_{0}\left\{1+\frac{Y}{\sqrt{v_{0}^{2}-4}}\right\}^{-\lambda_{+} v_{1} \sqrt{D}}
$$

and hence

$$
\beta \sim C_{0} e^{\lambda_{+} \sqrt{D} t Y}\left[\left\{1+\frac{Y}{\sqrt{v_{0}^{2}-4}}\right\}^{-\lambda_{+} v_{1} \sqrt{D}}+o(1)\right] \quad \text { as } t \rightarrow \infty \text { with } Y=O(1)
$$

This solution matches with the solution (23c) (which is uniform for $x \gg t$ as $t \rightarrow \infty$ ) in region A for $g(x) \in G_{2}$ as $Y \rightarrow \infty$, provided that

$$
\begin{aligned}
v_{0}=\sigma \sqrt{D}+1 / \sigma \sqrt{D}, \quad v_{1}=-n / \sigma \sqrt{D}, & C_{0}\left(a_{0}\right)=k_{0}\left(v_{0}^{2}-4\right)^{-n / 2} \\
& \text { for } 0<\sigma<1 / \sqrt{D},
\end{aligned}
$$

so that the constants $v_{0}, v_{1}$, and $a_{0}$ are now determined, since the function $C_{0}\left(a_{0}\right)$ is determined in region $I$. We note that the restriction $0<\sigma<1 / \sqrt{D}$ arises because 
we have specified the positive root of $\left(v_{0}^{2}-4\right)$ in the definition of the constant $\lambda_{+}$. This completes the asymptotic solution of the initial value problem (6), (7) as $t \rightarrow \infty$ for $g(x)=O\left(e^{-\sigma x} x^{-n}\right)$ as $x \rightarrow \infty$, with $0<\sigma<1 / \sqrt{D},-\infty<n<\infty$. We can summarize the leading order asymptotic solution in each region as:

RegION IIa. $x=O(1)$,

$$
\begin{aligned}
& \alpha=t^{-v_{1} \sqrt{D} \mu_{+}} e^{-v_{0} \sqrt{D} \mu_{+} t} \hat{a}_{0} \cosh \mu_{+} x+o\left(t^{-v_{1} \sqrt{D} \mu_{+}} e^{-v_{0} \sqrt{D} \mu_{+} t}\right) \\
& \beta=1+\hat{b}_{0}(D t)^{-1 / 2}+o\left((D t)^{-1 / 2}\right) \quad \text { as } t \rightarrow \infty \text { with } x=O(1) .
\end{aligned}
$$

REgION IIb. $x=O\left(t^{1 / 2}\right)$,

$$
\begin{gathered}
\alpha=a_{0} t^{-v_{1} \sqrt{D} \mu_{+}} e^{-v_{0} \sqrt{D} \mu_{+} t} \exp \left\{t^{1 / 2} \mu_{+} \sqrt{D} \bar{x}-\frac{\hat{b}_{0} \sqrt{\pi D}}{2 \mu_{+}} \operatorname{erfc}\left(\frac{\bar{x}}{2}\right)+o(1)\right\}, \\
\beta=1+\hat{b}_{0} e^{-\bar{x}^{2} / 4}(D t)^{-1 / 2}+o\left((D t)^{-1 / 2}\right) \\
\text { as } t \rightarrow \infty \text { with } \bar{x}=O(1), \text { where } \bar{x}=x /(D t)^{1 / 2} .
\end{gathered}
$$

Region I. $x=v_{0} \sqrt{D} t+v_{1} \sqrt{D} \log t+O(1), \quad \alpha=\alpha^{*}(y)+o(1)$,

$$
\beta=\beta^{*}(y)+o(1) \quad \text { at } t \rightarrow \infty \text { with } y=O(1),
$$

where $y=x-\sqrt{D} \gamma(t), \gamma(t) \sim v_{0} t+v_{1} \log t+o(\log t)$ as $t \rightarrow \infty$, and $\alpha^{*}(y), \beta^{*}(y)$ is the permanent form travelling wave solution of equations (6) with propagation speed $v_{0} \sqrt{D}$.

REGION III. $x=v_{0} \sqrt{D} t+v_{1} \sqrt{D} \log t+O(t), \quad \alpha=1+o\left(t^{-m}\right) \quad \forall m>0$,

$$
\begin{aligned}
\beta=k_{0} e^{-\sigma \sqrt{D} t Y}\left[\left\{\sqrt{v_{0}^{2}-4}+Y\right\}^{-n}+o(1)\right] \quad \text { as } t \rightarrow \infty \\
\text { with } Y=O(1), \text { where } Y=\{x-\sqrt{D} \gamma(t)\} / D^{1 / 2} t .
\end{aligned}
$$

The final region $\mathrm{A}$ is again passive and simply transmits the solution in region III, which remains uniform as $Y \rightarrow \infty$.

In regions IIa and IIb the reactant, $A$, is effectively completely consumed, whilst the concentration of the autocatalyst, $B$, diffuses down to unity as $t \rightarrow \infty$. Region I encompasses a travelling wave with propagation speed $v(t)=\sqrt{D} d \gamma / d t \sim$ $(D \sigma+1 / \sigma)-(n / \sigma) t^{-1}$ as $t \rightarrow \infty$. The qualitative form of this travelling wave solution for various $D$ and $\sigma$ was studied in [2] and is not examined here. In this case, the travelling wave propagates at a speed greater than the minimum, $2 \sqrt{D}$. This is because the form of the initial input functions $g(x)$ is such that initially there is sufficient autocatalyst present for the chemical reaction to remain significant as $x \rightarrow \infty$ and the balance between reaction and diffusion generates a fast wave. Fially, region III allows the small concentration $\beta$ as $Y \rightarrow \infty$ to take on the precise form in the far-field generated by the initial conditions in region A. The numerical 


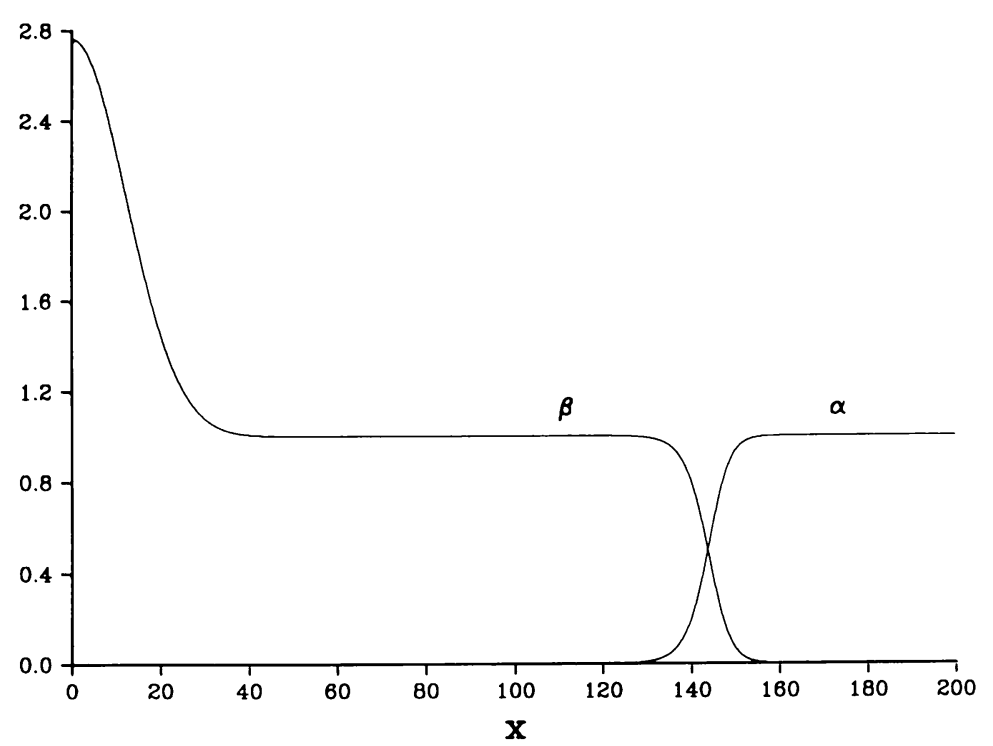

Fig. 2(a). A graph of the solution of the initial value problem (6), (7) when $D=1$ and $g(x)$ is given by Eq. (63) (on p. 370) with $\sigma=0.5$ and $n=-2$, at $t=50$.

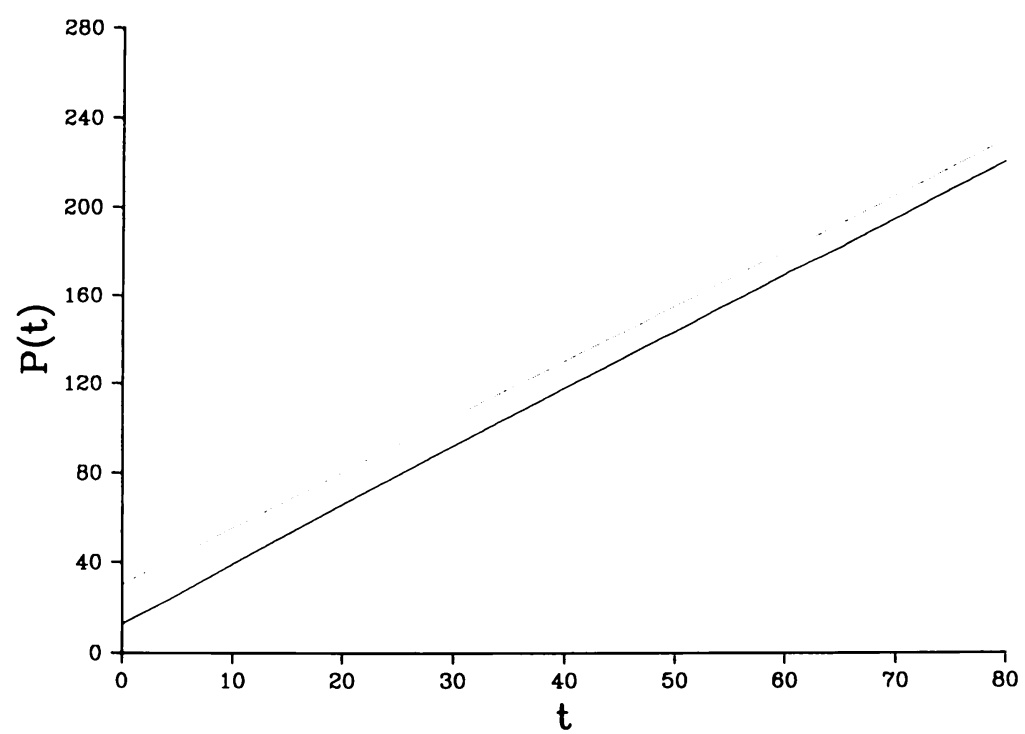

FIG. 2(b). A graph of the function $P(t)$. The broken line has gradient $(D \sigma+1 / \sigma)=2.5$ in this case.

solution of the initial value problem (6), (7) is illustrated in Fig. 2(a) in the case $D=1, \sigma=0.5, n=-2$, at $t=50$ and clearly shows the features described above. Numerical solutions of the initial value problem (6), (7) are discussed in detail in Sec. 3. We now turn to the case when $v_{0}=2$.

2.2.2. $v_{0}=2$. In this case appropriate scaled variables for region III are, from 


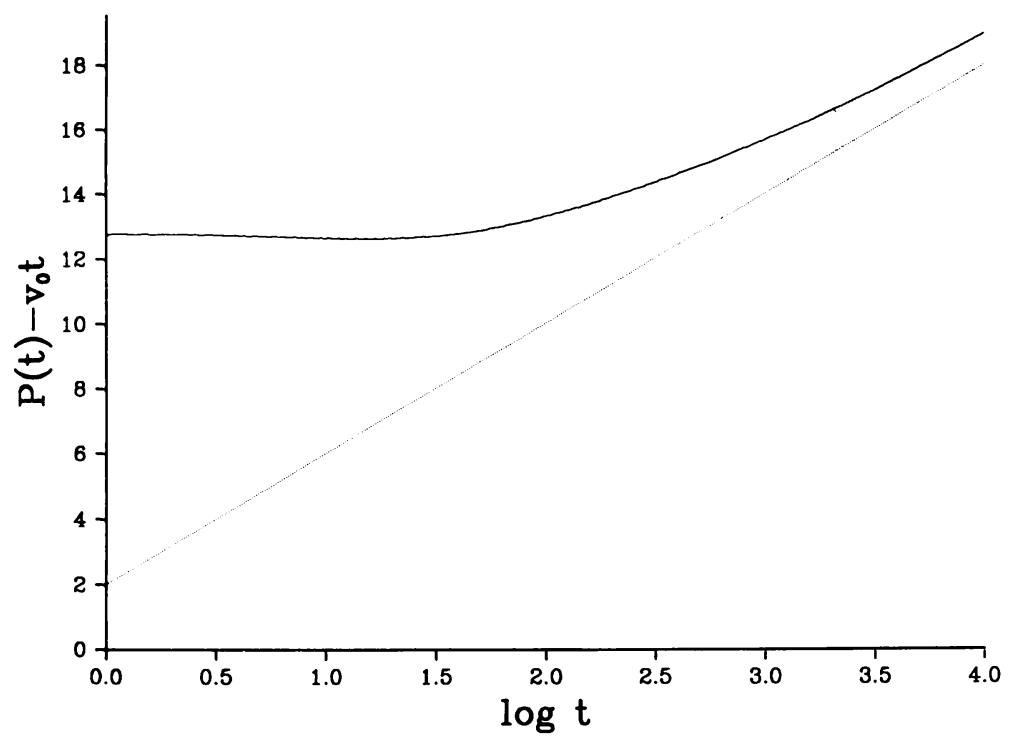

Fig. 2(c). A graph of the function $P(t)-(D \sigma+1 / \sigma) t$ plotted against $\log t$. The broken line has gradient $-n / \sigma=4$ in this case.

expansions (38),

$$
\begin{array}{ll}
\eta=y /(D t)^{1 / 2}, \quad \alpha=1+o\left(t^{-m}\right) \quad \forall m>0, \quad \beta=t^{1 / 2} e^{-t^{1 / 2} \eta} f \\
& \text { as } t \rightarrow \infty \text { with } \eta=O(1) \text { and } \eta>0,
\end{array}
$$

in terms of which Eq. (6b) becomes

$$
f_{\eta \eta}+\frac{1}{2} \eta f_{\eta}-\left(v_{1}+\frac{1}{2}\right) f+t^{-1 / 2} v_{1} f_{\eta}+o\left(t^{-1 / 2} f_{\eta}\right)=t f_{t} .
$$

This equation is to be solved subject to matching with expansion (38) in region I as $\eta \rightarrow 0$, and with the solution (23c) or (26b) in region $\mathrm{A}$ as $\eta \rightarrow \infty$. In addition we require $f(\eta)>0$ for $\eta>0$. We expand $f$ as

$$
f=f_{0}+t^{-1 / 2} f_{1}+o\left(t^{-1 / 2}\right) \quad \text { as } t \rightarrow \infty \text { with } \eta=O(1),
$$

and substitute into Eq. (42b). At leading order, we obtain the equation

$$
f_{0}^{\prime \prime}+\frac{1}{2} \eta f_{0}^{\prime}-\left(v_{1}+\frac{1}{2}\right) f_{0}=0
$$

which is to be solved subject to the matching condition

$$
f_{0} \sim A_{0}\left(a_{0}\right) \sqrt{D} \eta\left(1+v_{1} \eta^{2} / 6\right) \text { as } \eta \rightarrow 0 \quad \text { and } \quad f(\eta)>0 \quad \forall \eta>0 .
$$

Now, the solution of Eq. (43a), which has $f_{0} \sim A_{0} \sqrt{D} \eta$ as $\eta \rightarrow 0$, can be written in terms of confluent hypergeometric functions [15] as

$$
f_{0}(\eta)=A_{0} \sqrt{D} \eta e^{-\eta^{2} / 4} F_{1}\left(\frac{3}{2}+v_{1} ; \frac{3}{2} ; \frac{\eta^{2}}{4}\right) .
$$


First, we note from the properties of ${ }_{1} F_{1}$ that $f_{0}$ becomes negative for some finite $\eta>0$ for all $v_{1}<-3 / 2$. Therefore the solution (44a) does not satisfy the second of conditions $(43 \mathrm{~b})$ in this case and we conclude that $v_{1} \geq-3 / 2$. Now, by considering the behaviour of the confluent hypergeometric function ${ }_{1} F_{1}$ as $\eta \rightarrow \infty$, we find that

$$
\begin{array}{ll}
f_{0} \sim \frac{A_{0} \sqrt{\pi D}}{\left(v_{1}+\frac{1}{2}\right) ! 2^{2 v_{1}+1}} \eta^{2 v_{1}+1} & \text { for } \eta \gg 1 \text { with } v_{1}>-\frac{3}{2}, \\
f_{0}=A_{0} \sqrt{D} \eta e^{-\eta^{2} / 4} & \text { for } v_{1}=-\frac{3}{2} .
\end{array}
$$

As $\eta \rightarrow \infty$, we therefore have that $f_{0}$ behaves algebraically for $v_{1}>-\frac{3}{2}$ whereas $f_{0} \rightarrow 0$ exponentially for $v_{1}=-\frac{3}{2}$. Before considering these two cases separately, we determine the next term in the expansion (42c).

At $O\left(t^{-1 / 2}\right)$, Eq. $(42 \mathrm{~b})$ becomes

$$
f_{1}^{\prime \prime}+\frac{1}{2} \eta f_{1}^{\prime}-v_{1} f_{1}=-v_{1} f_{0}^{\prime},
$$

which is to be solved subject to the matching conditions

$$
f_{1} \sim B_{0}-\frac{b_{1} A_{0}}{\sqrt{D}} \eta+\frac{1}{2} v_{1}\left(B_{0}-A_{0} \sqrt{D}\right) \eta^{2}, \quad \text { as } \eta \rightarrow 0 .
$$

After noting that $f_{1}=f_{0}^{\prime}$ is a solution of the homogeneous part of Eq. (45a) we can readily obtain the solution which satisfies the matching condition $(45 \mathrm{~b})$ (via variation of parameters) as

$$
\begin{aligned}
f_{1}= & -v_{1} f_{0}^{\prime}(\eta) \int_{0}^{\eta} \frac{e^{-s^{2} / 4}}{f_{0}^{\prime}(s)^{2}} \int_{0}^{s} f_{0}^{\prime}(u)^{2} e^{u^{2} / 4} d u d s \\
& -b_{1} A_{0}^{2} f_{0}^{\prime}(\eta) \int_{0}^{\eta} \frac{e^{-s^{2} / 4}}{f_{0}^{\prime}(s)^{2}} d s+\frac{B_{0} f_{0}^{\prime}(\eta)}{A_{0} \sqrt{D}} .
\end{aligned}
$$

We now consider the cases $v_{1}>-\frac{3}{2}$ and $v_{1}=-\frac{3}{2}$ separately.

(i) $v_{1}>-\frac{3}{2}$. In this case we find that

$$
f_{1} \sim-\frac{v_{1}\left(v_{1}+\frac{1}{2}\right) A_{0} \sqrt{\pi D}}{\left(v_{1}+\frac{1}{2}\right) ! 2^{2 v_{1}-1}} \eta^{2 v_{1}} \log \eta \quad \text { for } \eta \gg 1 .
$$

Expansion (42c) therefore shows that

$$
\begin{aligned}
f \sim \frac{A_{0} \sqrt{\pi D} 2 v_{1}}{\left(v_{1}+\frac{1}{2}\right) ! 2^{2 v_{1}+1}} \eta^{2 v_{1}+1}-t^{-1 / 2} \frac{v_{1}\left(v_{1}+\frac{1}{2}\right) A_{0} \sqrt{\pi D}}{\left(v_{1}+\frac{1}{2}\right) ! 2^{2 v_{1}-1}} \eta^{2 v_{1}} \log \eta+o\left(t^{-1 / 2}\right) \\
\text { as } t \rightarrow \infty \text { for } \eta \gg 1 .
\end{aligned}
$$

This expansion remains uniform as $\eta \rightarrow \infty$, and matches with the solution (23c) (which is uniform for $x \gg t$ as $t \rightarrow \infty$ ) in region A for $g(x) \in G_{2}$ provided that

$$
v_{1}=-\frac{1}{2}(n+1), \quad A_{0}\left(a_{0}\right)=\frac{\left(v_{1}+\frac{1}{2}\right) ! 2^{2 v_{1}+1} k_{0}}{\sqrt{\pi D}} \quad \text { for } \sigma=1 / \sqrt{D}, n<2,
$$




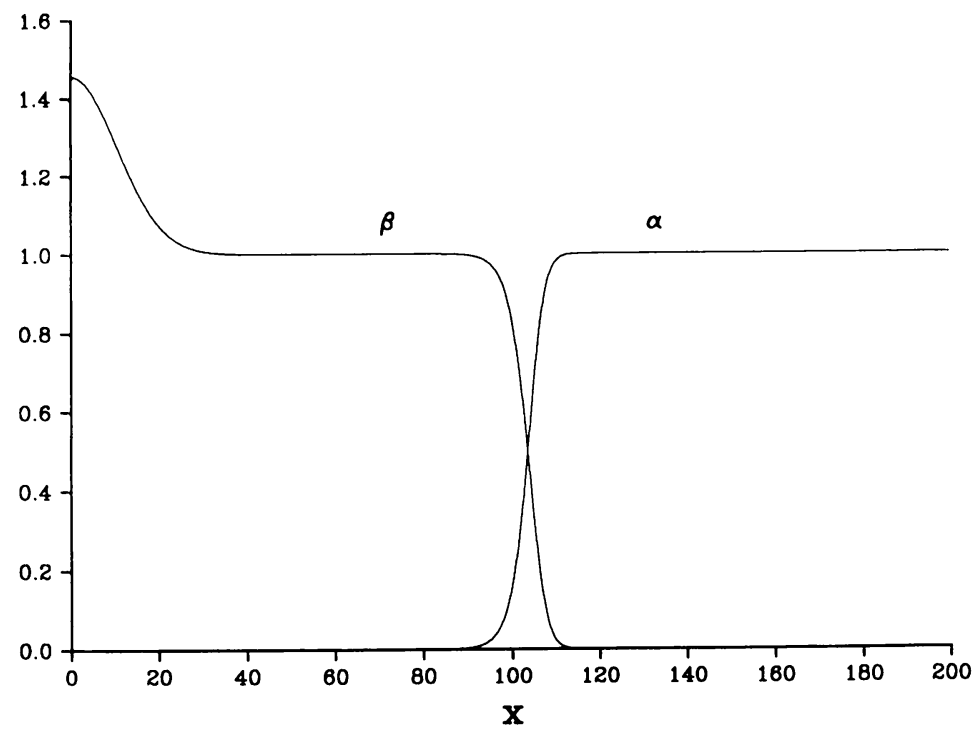

FIG. 3(a). A graph of the solution of the initial value problem (6), (7) when $D=1$ and $g(x)$ is given by Eq. (63) with $\sigma=1$ and $n=-2$, at $t=50$.

which fixes the constants $v_{1}$ and $a_{0}$. This completes the asymptotic solution of the initial value problem (6), (7), as $t \rightarrow \infty$ for $g(x)=O\left(e^{-x / \sqrt{D}} x^{-n}\right)$ as $x \rightarrow \infty$ with $n<2$. The leading order behaviour of the solution in regions I, IIa, and IIb is again given by $(41 \mathrm{a}, \mathrm{c}, \mathrm{b})$. However, we now have

REGION III. $x=2 \sqrt{D} t+v_{1} \sqrt{D} \log t+O\left(t^{1 / 2}\right), \quad \alpha=1+o\left(t^{-m}\right) \quad \forall m>0$,

$$
\begin{aligned}
\beta=t^{1 / 2} e^{-t^{1 / 2} \eta} k_{0}\left\{\eta^{-n}+o(1)\right\} \quad \text { as } t \rightarrow \infty & \text { with } \eta=O(1), \\
\text { where } \eta & =\{x-\sqrt{D} \gamma(t)\} /(D t)^{1 / 2} .
\end{aligned}
$$

Region $\mathrm{A}$ is again passive and simply transmits the solution in region III, which remains uniform as $\eta \rightarrow \infty$. Region I now encompasses a travelling wave with propagation speed $v(t) \sim 2 \sqrt{D}-\frac{1}{2}(n+1) \sqrt{D} t^{-1}$ as $t \rightarrow \infty$. In this case, the travelling wave propagates at the minimum speed as $t \rightarrow \infty$, but the correction to this speed is greater than the minimum with $v_{1}>-\frac{3}{2}$. Since the asymptotic form of the travelling wave speed depends upon $n$, we conclude that the form of the initial input function $g(x)$ is such that the chemical reaction is significant as $x \rightarrow \infty$. Finally, region III allows the small concentration $\beta$ as $\eta \rightarrow \infty$ to take on the form in the far-field generated by the initial conditions through region $\mathrm{A}$. The numerical solution of the initial value problem (6), (7) is illustrated in Fig. 3(a) in the case $D=1, \sigma=1, n=-2$ at $t=50$ and clearly displays the features described above. We now turn to the case $v_{1}=-\frac{3}{2}$.

(ii) $v_{1}=-\frac{3}{2}$. First we note that, by a process of elimination, all the cases where $g(x) \in G_{2}$ that have not already been considered (i.e., $\sigma=1 / \sqrt{D}, n \geq 2$ and $\sigma>1 / \sqrt{D},-\infty<n<\infty)$ and the case $g(x) \in G_{3}$ must have $v_{0}=2$ and $v_{1}=-\frac{3}{2}$. 


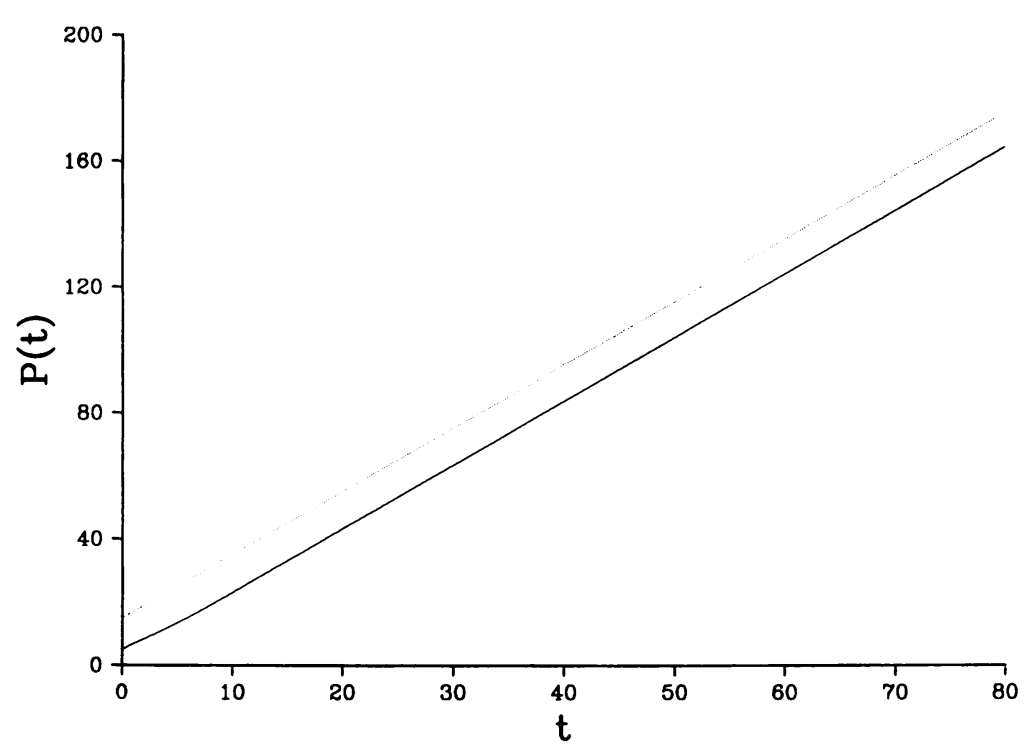

FIG. 3(b). A graph of the function $\log P(t)$. The broken line has gradient $2 \sqrt{D}=2$ in this case.

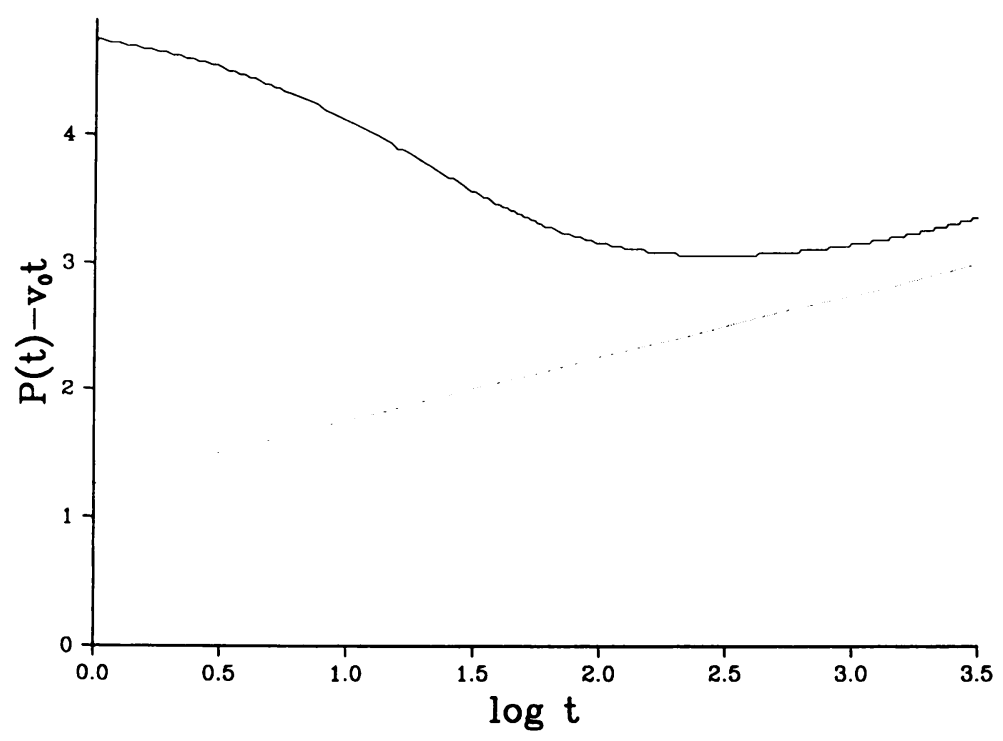

Fig. 3(c). A graph of the function $P(t)-2 \sqrt{D} t$ plotted against $\log t$. The broken line has gradient $-\frac{1}{2}(n+1) \sqrt{D}=\frac{1}{2}$ in this case.

When $v_{1}=-\frac{3}{2}$ we find that, in general, $f_{1} \rightarrow 0$ algebraically as $\eta \rightarrow \infty$. However, since in this case $f_{0}=A_{0} \sqrt{D} \eta e^{-\eta^{2} / 4}$, we must eliminate this algebraic decay of $f_{1}$, which would otherwise induce an exponential nonuniformity in expansion (42c) as $\eta \rightarrow \infty$. This is achieved by choosing

$$
b_{1}=3 D \sqrt{\pi},
$$


after which we find that

$$
\begin{aligned}
f_{1}= & -3 A_{0} \sqrt{D}\left(1-\frac{1}{2} \eta^{2}\right) e^{-\eta^{2} / 4} \int_{0}^{\eta} \frac{e^{s^{2} / 4}}{\left(1-\frac{1}{2} s^{2}\right)^{2}} \int_{s}^{\infty} e^{-u^{2} / 4} d u d s \\
& +\left\{B_{0}-\frac{1}{2}\left(B_{0}+3 A_{0} \sqrt{D}\right) \eta^{2}\right\} e^{-\eta^{2} / 4} \\
& -\frac{3}{2} A_{0} \sqrt{D}\left(1-\frac{1}{2} \eta^{2}\right) e^{-\eta^{2} / 4} \log \left(1-\frac{1}{2} \eta^{2}\right),
\end{aligned}
$$

and hence,

$$
f_{1} \sim\left[\frac{3}{2} A_{0} \sqrt{D} \log \eta-\frac{1}{2}\left\{B_{0}+3\left(1+\frac{1}{2} \log 2\right) A_{0} \sqrt{D}\right\}\right] \eta^{2} e^{-\eta^{2} / 4} \quad \text { for } \eta \gg 1 .
$$

This demonstrates the need for terms of $O\left(t^{-1 / 2}\right)$ in expansions (28a). From expansion $(42 \mathrm{c})$ we now find that

$$
\begin{aligned}
f \sim & A_{0} \sqrt{D} \eta e^{-\eta^{2} / 4}+t^{-1 / 2}\left[\frac{3}{2} A_{0} \sqrt{D} \log \eta-\frac{1}{2}\left\{B_{0}+3\left(1+\frac{1}{2} \log 2\right) A_{0} \sqrt{D}\right\}\right] \eta^{2} e^{-\eta^{2} / 4} \\
& +o\left(t^{-1 / 2}\right) \quad \text { as } t \rightarrow \infty \text { for } \eta \gg 1 .
\end{aligned}
$$

This expansion becomes nonuniform as $\eta \rightarrow \infty$, in particular when $\eta=O\left(t^{1 / 2}\right)$, and we must introduce a further asymptotic region in order to continue the solution when $\eta=O\left(t^{1 / 2}\right)$. Appropriate scaled variables for region IV are, from expansion $(51)$,

$$
\begin{aligned}
Y=y / D^{1 / 2} t, \quad \alpha=1+o\left(t^{-m}\right) \quad \forall m>0, \quad \beta=t^{1 / 2} e^{-t Y} e^{-t F(Y, t)} \\
\text { as } t \rightarrow \infty \text { with } Y=O(1) \text { and } Y>0,
\end{aligned}
$$

in terms of which Eq. (6b) becomes

$$
F_{Y}^{2}-Y F_{Y}+F+t^{-1}\left(1+\frac{3}{2} F_{Y}-F_{Y Y}\right)+t F_{t}+o\left(t^{-1} F_{Y}\right)=0 .
$$

We now expand $F$ as

$$
\begin{aligned}
& F=F_{0}(Y)+t^{-1} \log t F_{1}(Y)+t^{-1} F_{2}(Y)+o\left(t^{-1}\right) \\
& \text { as } t \rightarrow \infty \text { with } Y=O(1),
\end{aligned}
$$

and substitute into Eq. (52b). At leading order we obtain

$$
F_{0}^{\prime 2}-Y F_{0}^{\prime}+F_{0}=0
$$

which is to be solved subject to the matching condition

$$
F_{0} \sim Y^{2} / 4 \quad \text { as } Y \rightarrow 0 .
$$

Equation (53a) has a one-parameter family of linear solutions,

$$
F_{0}=c Y-c^{2},
$$

where $c$ is an arbitrary constant and the associated envelope solution

$$
F_{0}=Y^{2} / 4 \text {. }
$$




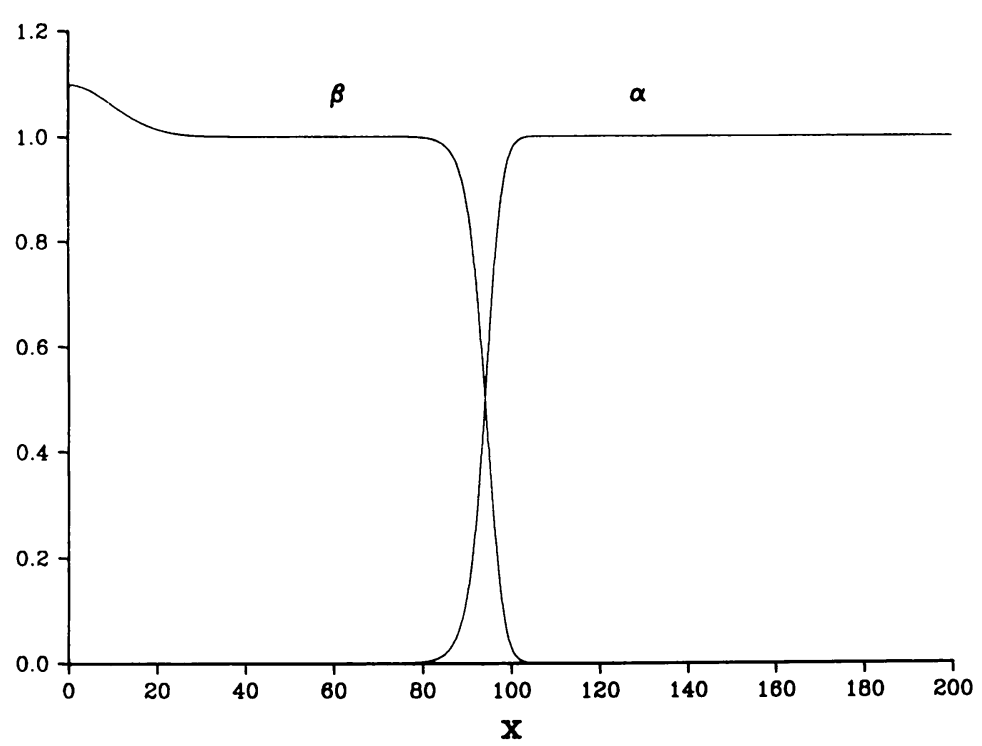

FIG. 4(a). A graph of the solution of the initial value problem (6), (7) when $D=1$ and $g(x)$ is given by Eq. (63) with $\sigma=1$ and $n=4$, at $t=50$.

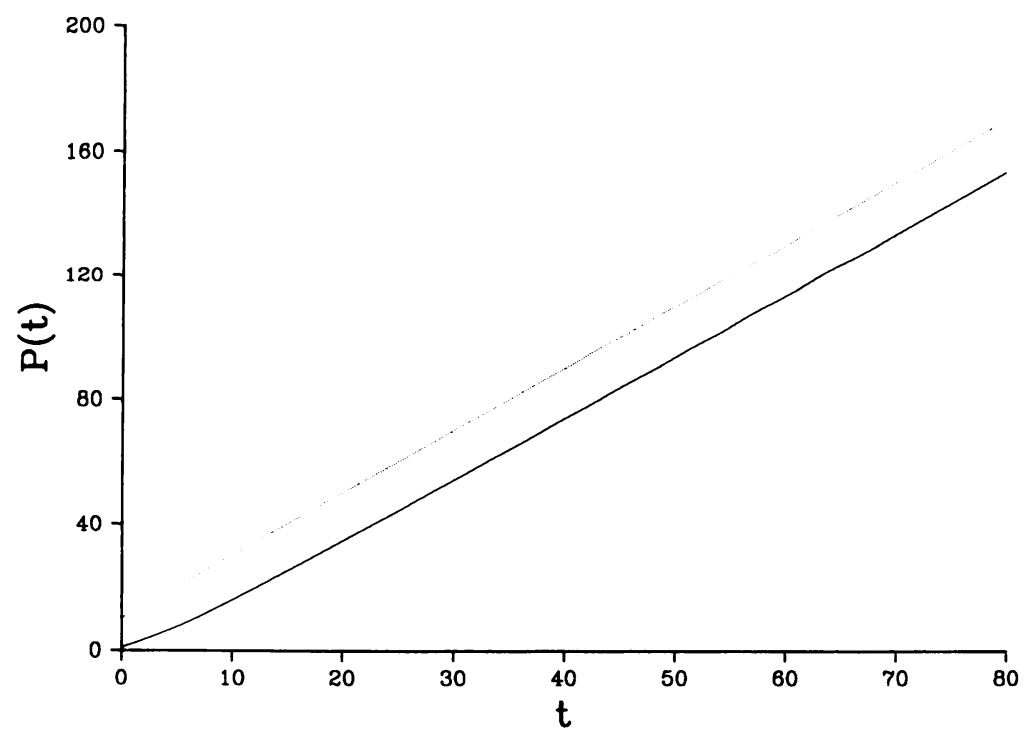

Fig. 4(b). A graph of the function $P(t)$. The broken line has gradient $2 \sqrt{D}=2$ in this case.

Therefore, we take the solution $F_{0}=Y^{2} / 4$, which satisfies the matching condition (53b) as $Y \rightarrow 0$. On continuing our analysis of the equations that arise when we substitute expansion (52c) into Eq. (52b) we obtain the solution at $O\left(t^{-1} \log t\right)$,

$$
F_{1}=-\frac{1}{2}-\frac{3}{4} Y
$$




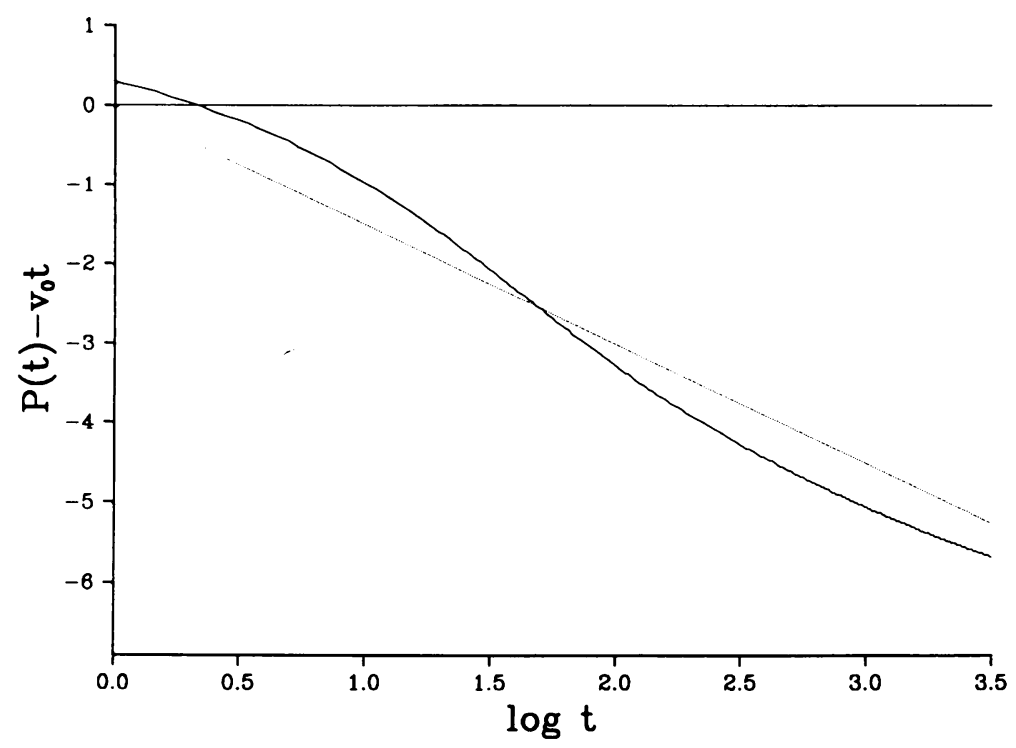

FIG. 4(c). A graph of the function $P(t)-2 \sqrt{D} t$ plotted against $\log t$. The broken line has gradient $-\frac{3}{2} \sqrt{D}=-\frac{3}{2}$ in this case.

which matches with expansion $(51)$ as $Y \rightarrow 0$. However, the function $F_{2}$ remains undetermined, and we conclude either that $F_{2}$ depends upon the detailed form of the initial input function $g(x)$ and hence cannot be determined by a large time asymptotic solution, or that $F_{2}$ is fixed by an equation that arises when we include exponentially small terms in the expansion (52c). The problem of determining $F_{2}$ is not pursued further in this paper. Expansion $(52 \mathrm{c})$ now becomes

$$
F=Y^{2} / 4-\left(\frac{1}{2}+\frac{3}{4} Y\right) t^{-1} \log t+O\left(t^{-1}\right) \quad \text { as } t \rightarrow \infty \text { for } Y=O(1) \text {. }
$$

This expansion matches with the solution (26c) (which is uniform for $x \gg t$ as $t \rightarrow \infty)$ in region $\mathrm{A}$ as $Y \rightarrow \infty$, when $g(x) \in G_{3}$ and completes the asymptotic solution of the initial value problem (6), (7) as $t \rightarrow \infty$ for $g(x)$ with compact support. The leading order behaviour of the solution in regions I, IIa, and IIb is again given by $(41 \mathrm{a}, \mathrm{b}, \mathrm{c})$, and we now have

REGION III. $x=2 \sqrt{D} t-\frac{3}{2} \sqrt{D} \log t+O\left(t^{1 / 2}\right), \quad \alpha=1+o\left(t^{-m}\right) \quad \forall m>0$,

$$
\begin{aligned}
\beta=t^{1 / 2} e^{-t^{1 / 2} \eta} A_{0} \sqrt{D} \eta e^{-\eta^{2} / 4}(1+o(1)) & \text { as } R t \rightarrow \infty \text { with } \eta=O(1), \\
& \text { where } \eta=\{x-\sqrt{D} \gamma(t)\} /(D t)^{1 / 2}
\end{aligned}
$$

$$
\text { REGION IV: } x=2 \sqrt{D} t-\frac{3}{2} \sqrt{D} \log t+O(t), \quad \alpha=1+o\left(t^{-m}\right) \quad \forall m>0,
$$

$$
\begin{array}{ll}
\beta=t^{1+(3 / 4) Y} e^{-t\left(Y+Y^{2} / 4+o(1)\right)} & \text { as } t \rightarrow \infty \text { with } Y=O(1), \\
& \text { where } Y=\{x-\sqrt{D} \gamma(t)\} / D^{1 / 2} t .
\end{array}
$$

Region $\mathrm{A}$ is again passive and simply transmits the solution in region IV which remains uniform as $Y \rightarrow \infty$. Region I now contains a travelling wave with the minimum propagation speed $v(t) \sim 2 \sqrt{D}-\frac{3}{2} \sqrt{D} t^{-1}$ as $t \rightarrow \infty$. This travelling wave 
represents a balance between reaction and diffusion with $\beta=O\left(y e^{-y / \sqrt{D}}\right)$ in region I as $y \rightarrow \infty$, which changes via regions III and IV to a diffusion-controlled solution, with $\beta=O\left(e^{-t Y^{2} / 4}\right)$ in region IV as $Y \rightarrow \infty$. The numerical solution of the initial value problem (6), (7) is illustrated in Fig. $6($ a) on p. 369 in the case $D=1, \lambda=1$, at $t=50$ and clearly displays the features described above.

We have now apparently exhausted all of the possibilities and have yet to deal with the case when $g(x) \in G_{2}$ and $\sigma>1 / \sqrt{D}$ with $-\infty<n<\infty$ or $\sigma=1 / \sqrt{D}$ with $n \geq 2$. However, we note that the solution (54a) matches with the solution (23c) (which is uniform for $x \gg t$ as $t \rightarrow \infty$ ) in region $\mathrm{A}$ as $Y \rightarrow \infty$ if $c=(\sigma \sqrt{D}-1$ ) with $\sigma>1 / \sqrt{D}$, and therefore, in order to satisfy the matching condition (53b) we require the leading order solution

$$
F_{0}= \begin{cases}Y^{2} / 4 & 0<Y<2(\sigma \sqrt{D}-1) \\ (\sigma \sqrt{D}-1) Y-(\sigma \sqrt{D}-1)^{2} & Y \geq 2(\sigma \sqrt{D}-1)\end{cases}
$$

Although $F_{0}$ and $F_{0}^{\prime}$ are continuous for $Y>0$, the second derivative $F_{0}^{\prime \prime}$ is discontinuous at the point $Y=2(\sigma \sqrt{D}-1)$. This indicates that a thin asymptotic transition region exists in the neighbourhood of the point $Y=2(\sigma \sqrt{D}-1)$ in which second derivatives are retained to smooth out this discontinuity in curvature. We can confirm this by calculating further terms in the expansion (52c) for $Y>2(\sigma \sqrt{D}-1)$, which shows that

$$
F=\left\{\begin{array}{c}
Y^{2} / 4-\left(\frac{1}{2}+\frac{3}{4} Y\right) t^{-1} \log t+O\left(t^{-1}\right) \quad 0<Y<2(\sigma \sqrt{D}-1), \\
\left\{(\sigma \sqrt{D}-1) Y-(\sigma \sqrt{D}-1)^{2}\right\}+\left\{n-1-\frac{3}{2}(\sigma \sqrt{D}-1)\right\} t^{-1} \log t \\
\quad+n \log \{Y-2(\sigma \sqrt{D}-1)\} t^{-1}+o\left(t^{-1}\right) \quad Y \geq 2(\sigma \sqrt{D}-1),
\end{array}\right.
$$

and $t \rightarrow \infty$ and hence expansion (58) becomes nonuniform in the neighbourhood of the point $Y=2(\sigma \sqrt{D}-1)$. The nature of this passive transition region is not pursued further in this paper. This completes the leading order asymptotic solution of the initial value problem (6), (7) as $t \rightarrow \infty$ for $g(x) \in G_{2}$ with $\sigma>1 / \sqrt{D}$, $-\infty<n<\infty$. The behaviour of the solution at leading order in regions I, IIa, IIb, and III is given by $(41 \mathrm{a}, \mathrm{b}, \mathrm{c})$ and (56a), and we now have

REGION IV. $x=2 \sqrt{D} t-\frac{3}{2} \sqrt{D} \log t+O(t), \quad \alpha=1+o\left(t^{-m}\right) \quad \forall m>0$,

$$
\beta=\left\{\begin{array}{c}
t^{1+(3 / 4) Y} e^{-t\left(Y+Y^{2} / 4+o(1)\right)} \quad 0<Y<2(\sigma \sqrt{D}-1)-o(1), \\
t^{((3 / 2) \sigma \sqrt{D}-n)} e^{-\sigma \sqrt{D} t Y} e^{(\sigma \sqrt{D}-1)^{2} t}\{Y-2(\sigma \sqrt{D}-1)\}^{-n} \\
Y>2(\sigma \sqrt{D}-1)+o(1) \quad \text { as } t \rightarrow \infty, \\
\text { where } Y=\{x-\sqrt{D} \gamma(t)\} / D^{1 / 2} t
\end{array}\right.
$$

Region A is passive and simply transmits the solution in region IV, which remains uniform as $Y \rightarrow \infty$. Region I again contains a travelling wave that propagates at the minimum speed $v(t) \sim 2 \sqrt{D}-\frac{3}{2} \sqrt{D} t^{-1}$ as $t \rightarrow \infty$, and represents a balance between reaction and diffusion. Regions III and IV accommodate the transition in the small concentration $\beta$ from the travelling wave profile, $\beta=O\left(y e^{-y / \sqrt{D}}\right)$ 


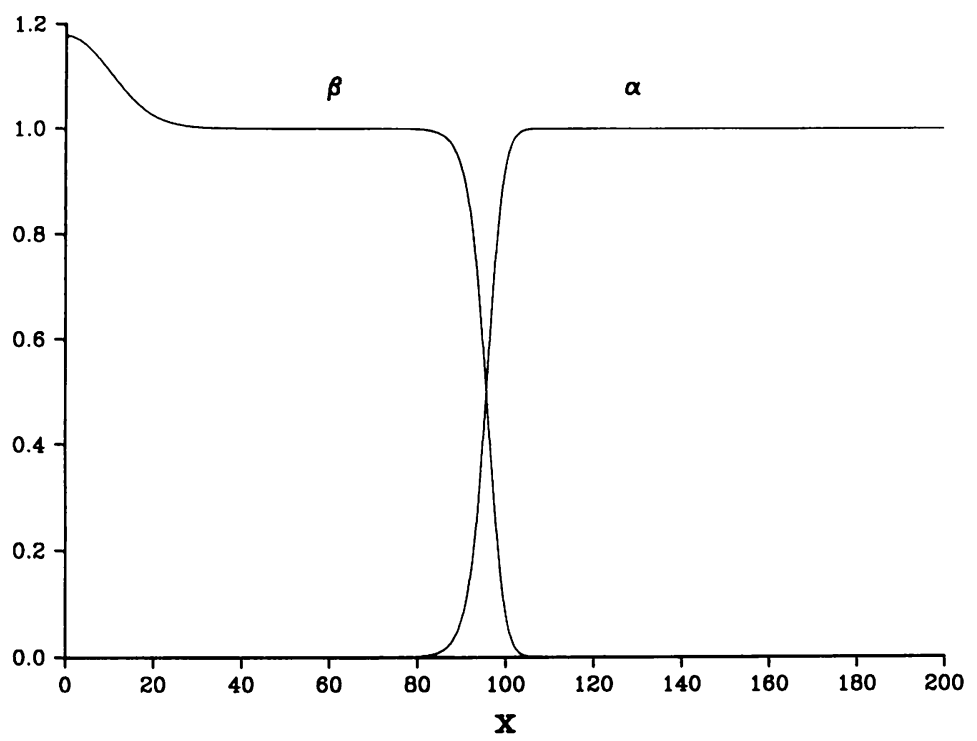

Fig. 5(a). A graph of the solution of the initial value problem (6), (7) when $D=1$ and $g(x)$ is given by Eq. (63) with $\sigma=2$ and $n=-2$, at $t=50$.

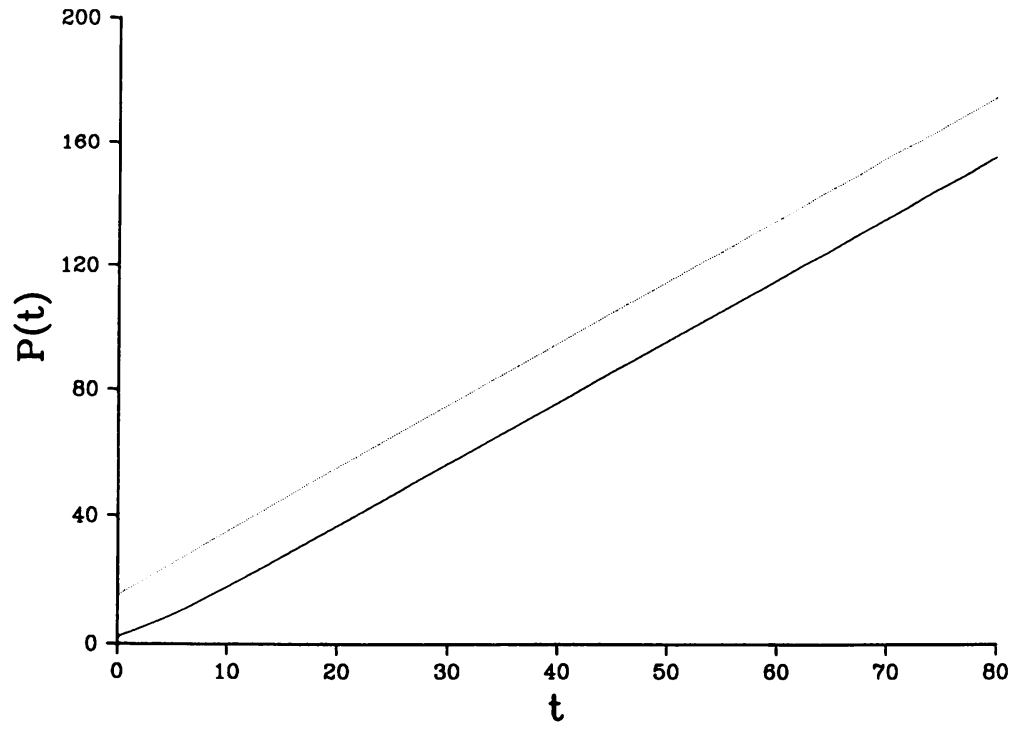

Fig. 5(b). A graph of the function $P(t)$. The broken line has gradient $2 \sqrt{D}=2$ in this case. 


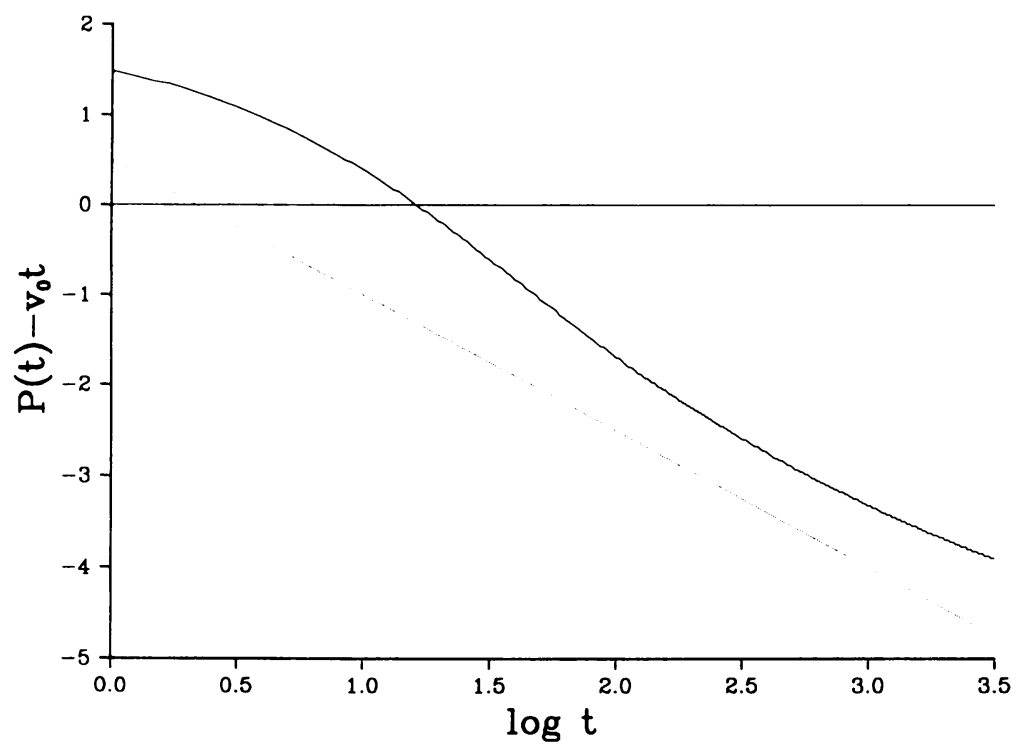

FIG. 5(c). A graph of the function $P(t)-2 \sqrt{D} t$ plotted against $\log t$. The broken line has gradient $-\frac{3}{2} \sqrt{D}=-\frac{3}{2}$ in this case.

as $y \rightarrow \infty$ in region $\mathrm{I}$, to the far-field determined by the initial input function, $\beta=O\left(t^{((3 / 2) \sigma \sqrt{D}-n)} e^{-\sigma \sqrt{D} t Y} e^{(\sigma \sqrt{D}-1)^{2} t} Y^{-n}\right)$ as $Y \rightarrow \infty$ in region IV. This transition is rapid in the neighbourhood of the point $Y=Y_{0}$, where $Y_{0}=2(\sigma \sqrt{D}-1)+o(1)$ as $t \rightarrow \infty$, or equivalently, $x=x_{0}$ where $x_{0}=2 \sigma D t+o(t)$ as $t \rightarrow \infty$. The numerical solution of the initial value problem (6), (7) is illustrated in Fig. 5(a) in the case $D=1, \sigma=2, n=-2$ at $t=50$ and clearly displays the features described above.

In the only remaining case, $g(x) \in G_{2}$ with $\sigma=1 / \sqrt{D}, n \geq 2$, our analysis of the solution in region IV indicates that a rapid transition from the envelope solution $F_{0}=Y^{2} / 4$ occurs in the neighbourhood of the point $Y=Y_{0}$, where now $Y_{0}=o(1)$ as $t \rightarrow \infty$. However, we know that the travelling wave propagation speed $v(t) \sim$ $2 \sqrt{D}-\frac{3}{2} \sqrt{D} t^{-1}$ as $t \rightarrow \infty$ in this case and we do not pursue the additional details of the solution further.

The conclusions of this section are summarized in Result 1.1 which we have now formally established via the method of matched asymptotic expansions.

3. Numerical solution of the initial value problem. In this section, we describe numerical solutions of the initial value problem (6), (7) for various values of $D$ and functions $g(x)$. We obtain these numerical solutions by the method described in detail in [3], which is closely related to the numerical method described in Merkin and Needham [12]. Briefly, we use a Crank-Nicolson method with a simple iterative procedure for solving the nonlinear algebraic equations which arise at each time step. A variable time step is also used in order to increase the efficiency of the numerical integration. When $0<D \ll 1$ the solution develops large gradients in the concentration $\beta$, as described in [3], which leads to problems with the accuracy of 
the numerical solutions. However, for moderate values of $D$ these problems do not arise. In addition to the concentrations $\alpha$ and $\beta$, we also calculated the function $P(t)$ at each time step, where $P(t)$ is defined by

$$
P(t)=\inf \left\{x: \beta(x, t)=\frac{1}{2}, x \geq 0\right\} .
$$

The function $P(t)$ provides a measure of the position of the wavefront in $\beta$ and hence $P(t) \sim \sqrt{D} \gamma(t)$ as $t \rightarrow \infty$.

Our aim in studying numerical solutions of the initial value problem (6), (7) is to verify that these solutions have the structure predicted by the asymptotic analysis for $t \gg 1$, which we described in Sec. 2. We do not therefore discuss the initial transient behaviour of the solution, which becomes quite intricate as $D \rightarrow 0$, see [3]. The numerical results which we present below are for the case $D=1$. We obtained similar results, in line with the predictions of Proposition 1.1, when $D \neq 1$.

(i) $g(x) \in G_{1}$. In this section, we study the solution when the initial conditions (7a) are

$$
\alpha(x, 0)=1, \quad \beta(x, 0)=\frac{1}{1+x^{n}} \quad \text { for } x \geq 0, n>1 .
$$

Fig. 1(a) shows the solution of the initial value problem when $D=1$ and $n=4$ at $t=20$. A phase wave is clearly visible, along with the remnant of the initial input of the autocatalyst, $B$, for $x=O(1)$, which diffuses down to unity as $t \rightarrow \infty$. The function $P(t)$ increases rapidly as $t$ increases. This is consistent with the analysis of Sec. 2.1, which shows that $k_{0}\left(k_{0}+P^{n} e^{-t}\right)^{-1} \sim \frac{1}{2}$ as $t \rightarrow \infty$, and hence

$$
\log P \sim t / n \quad \text { as } t \rightarrow \infty .
$$

The graph of $\log P$ plotted against $t$ is illustrated in Fig. 1(b) on p. 350 and shows that $\log P$ is asymptotic to a straight line with gradient $1 / n=\frac{1}{4}$ in this case, consistent with our analysis. We obtained similar results for other values of $n$.

(ii) $g(x) \in G_{2}$. In this section, we study the solution when the initial conditions are

$$
\alpha(x, 0)=1, \quad \beta(x, 0)= \begin{cases}1 & 0 \leq x \leq 1, \\ e^{-\sigma(x-1)} x^{-n} & x \geq 1, \sigma>0,-\infty<n<\infty .\end{cases}
$$

In this case we find that a travelling wave evolves, with $P(t) \sim \sqrt{D} \gamma(t) \sim v_{0} \sqrt{D} t+$ $v_{1} \sqrt{D} \log t$ as $t \rightarrow \infty$, where $v_{0}$ and $v_{1}$ are given by Result 1.1 . Figs. 2, 3, 4, and 5 show the solution at $t=50$, a graph of the function $P(t)$, and a graph of the function $P(t)-v_{0} \sqrt{D} t$ plotted against $\log t$ for various $\sigma$ and $n$. We find that both $P(t)$ and $P(t)-v_{0} \sqrt{D} t$ as a function of $\log t$ are asymptotic to straight lines with gradients $v_{0} \sqrt{D}$ and $v_{1} \sqrt{D}$, respectively, as $t \rightarrow \infty$ consistent with Result 1.1.

(iii) $g(x) \in G_{3}$. In this section, we study the solution when the initial conditions are

$$
\alpha(x, 0)=1, \quad \beta(x, 0)= \begin{cases}1 & 0 \leq x<1 \\ 0 & x \geq 1\end{cases}
$$


In this case we find that a travelling wave evolves, with $P(t) \sim \sqrt{D} \gamma(t) \sim 2 \sqrt{D} t-$ $\frac{3}{2} \sqrt{D} \log t$ as $t \rightarrow \infty$, consistent with Result 1.1. Figure 6(a) shows the solution for $D=1$ at $t=50$ which has evolved into a minimum speed travelling wave. Figures 6(b) and 6(c) show graphs of the functions $P(t)$ plotted against $t$ and $P(t)-2 t$ plotted against $\log t$, respectively. Both of these functions are asymptotic to straight lines as $t \rightarrow \infty$ with gradients 2 and $-\frac{3}{2}$, respectively, as predicted by Result 1.1.

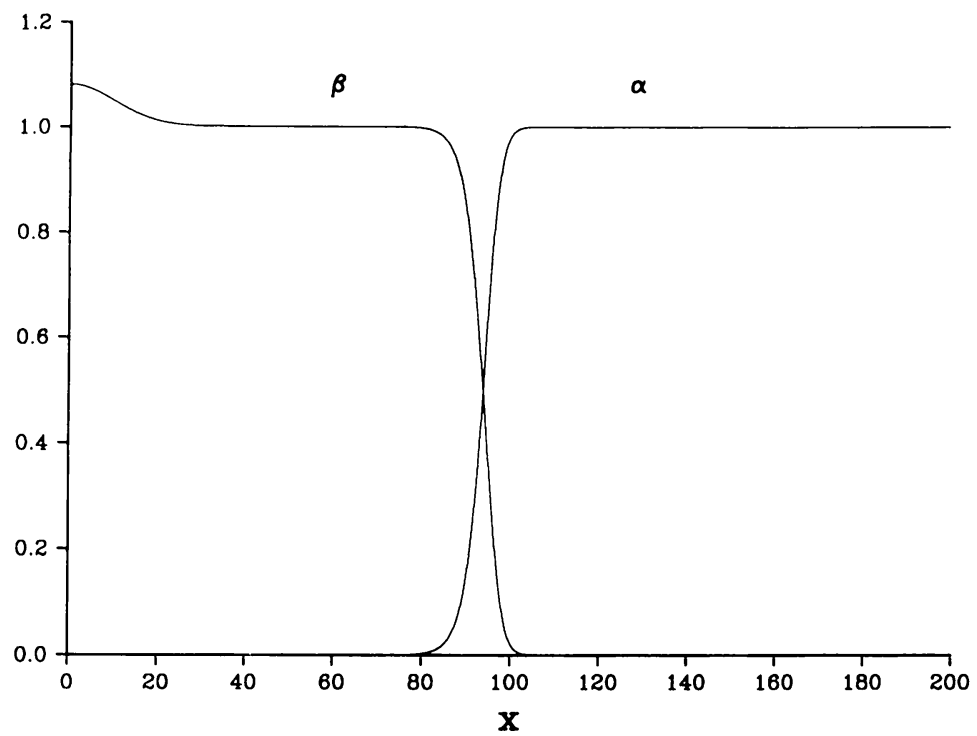

FIG. 6(a). A graph of the solution of the initial value problem (6), (7) when $D=1$ and $g(x)$ is given by Eq. (64) at $t=50$.

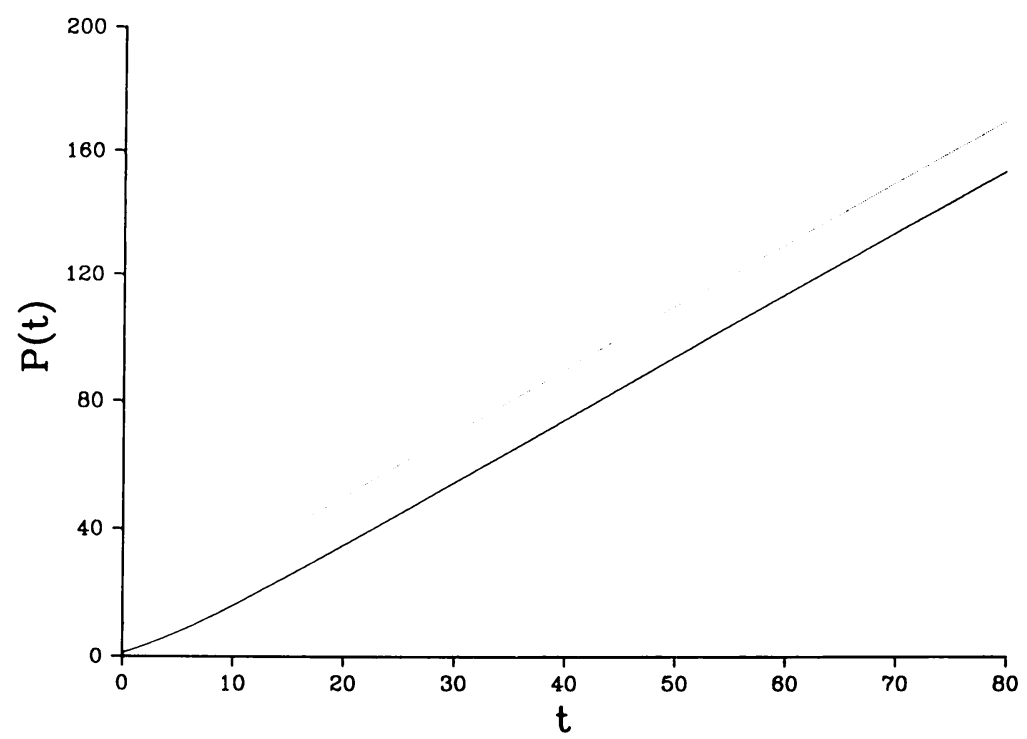

Fig. 6(b). A graph of the function $P(t)$. The broken line has gradient $2 \sqrt{D}=2$ in this case. 


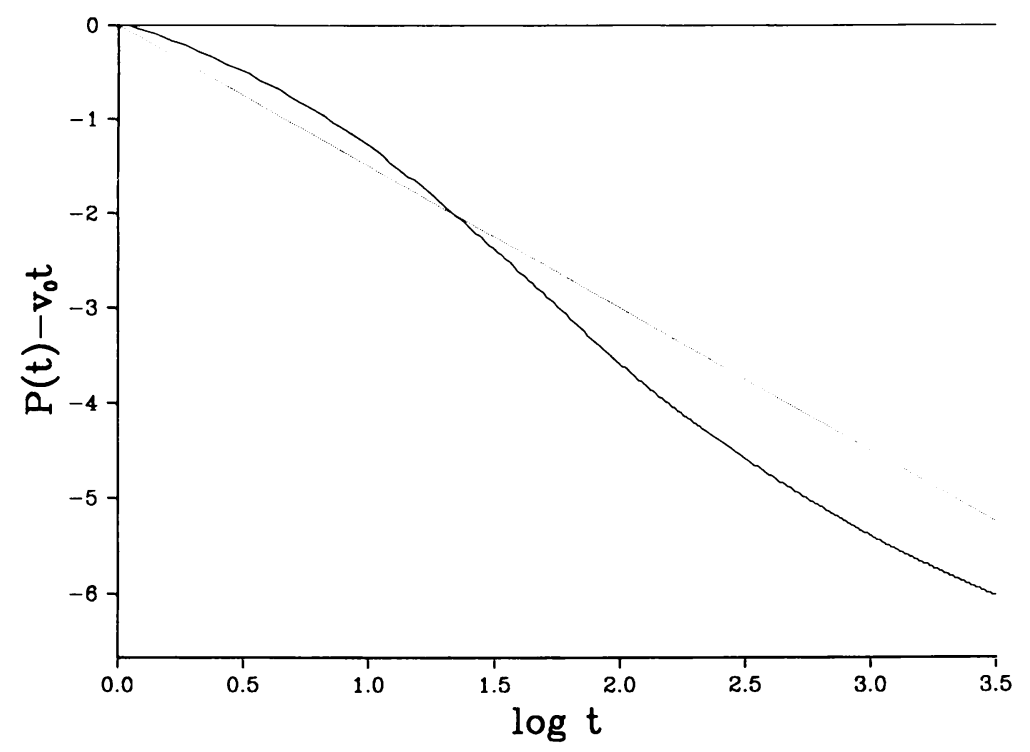

Fig. 6(c). A graph of the function $P(t)-2 \sqrt{D} t$ plotted against $\log t$. The broken line has gradient $-\frac{3}{2} \sqrt{D}=-\frac{3}{2}$ in this case.

4. Conclusion. The main conclusions of this paper are summarized in Result 1.1, which we now describe physically in terms of the competing processes of reaction and diffusion that tend to increase and decrease the concentration of the autocatalyst, $B$, respectively. When the initial input of the autocatalyst, $B$, decays sufficiently rapidly as $x \rightarrow \infty \quad\left(\beta(x, 0)=o\left(e^{-x \sqrt{D}} x^{-2}\right)\right.$ as $\left.x \rightarrow \infty\right)$, the travelling wave that evolves propagates at the minimum asymptotic speed, $v(t) \sim 2 \sqrt{D}-\frac{3}{2} \sqrt{D} t^{-1}$ as $t \rightarrow \infty$. This travelling wave pushes ahead of it a diffusion-dominated region (region IV) where a small concentration of $B$ initiates the autocatalytic production of $B$, which allows the travelling wave to propagate forward. The travelling wave in this case represents a balance between reaction and diffusion.

If the initial input of $B$ decays less rapidly, but still exponentially, as $x \rightarrow \infty$ $\left(\beta(x, 0)=O\left(e^{-\sigma x} x^{-n}\right)\right.$ as $x \rightarrow \infty$, with $\sigma=1 / \sqrt{D}, n<2$ or $\sigma<1 / \sqrt{D}$, $-\infty<n<\infty)$, the initial distribution of $B$ for $x \gg 1$ is sufficiently large to drive the evolution of a faster wave with $v(t)>2 \sqrt{D}-\frac{3}{2} \sqrt{D} t^{-1}$ as $t \rightarrow \infty$. Although diffusion still plays an essential part in the propagation of this faster travelling wave, the chemical reaction is the dominant process in this case. Finally, if the initial input of $B$ decays more slowly than any exponential as $x \rightarrow \infty\left(e^{\sigma x} \beta(x, 0) \rightarrow \infty\right.$ as $x \rightarrow \infty$ for all $\sigma>0$ ), a phase wave is generated. In this case, diffusion is negligible and the behaviour of the solution is completely dominated by the chemical reaction as $t \rightarrow \infty$.

Acknowledgment. The first author acknowledges the assistance of an SERC Research Studentship. 


\section{REFERENCES}

[1] N. J. T. Bailey, The Mathematical Theory of Infectious Diseases, Griffen, London, 1975

[2] J. Billingham and D. J. Needham, The development of travelling waves in quadratic and cubic autocatalysis with unequal diffusion rates. I Permanent form travelling waves, Philos. Trans. Roy. Soc. London Ser. A 334, 1-25 (1991).

[3] J. Billingham and D. J. Needham, The development of travelling waves in quadratic and cubic autocatalysis with unequal diffusion rates. II An initial value problem with an immobilized or nearly immobilized autocatalyst, to appear in Philos. Trans. Roy. Soc. London Ser A 336.

[4] M. D. Bramson, Maximal displacement of branching Brownian motion, Comm. Pure Appl. Math. 31, 531-581 (1978)

[5] R. J. Field and R. M. Noyes, Oscillations in chemical systems. IV Limit cycle behaviour in a model of a real chemical reaction, J. Chem. Phys. 60, 1877-1884 (1974)

[6] R. A. Fisher, The wave of advance of advantageous genes, Ann. of Eugenics 7, 355-369 (1937)

[7] P. Gray, J. F. Griffiths, and S. K. Scott, Oscillation, glow and ignition in carbon monoxide oxidation in an open system, Proc. Roy. Soc. London Ser. A 397, 21-44 (1984)

[8] A. Hanna, A. Saul, and K. Showalter, Detailed studies of propagating fronts in the iodate oxidation of arsenous acid, J. Amer. Chem. Soc. 104, 3838-3844 (1982)

[9] A. Kolmogorov, I. Petrovskii, and N. Piskounov, Etude de l'équation de la diffusion avec croissance de la quantité de matière et son application à un problème biologique, Moscow Univ. Math. Bull. 1, 1-25 (1937)

[10] D. A. Larson, Transient bounds and time-asymptotic behaviour of solutions to nonlinear equations of Fisher type, SIAM J. Appl. Math. 34, 93-103 (1978)

[11] H. P. McKean, Application of Brownian motion to the equation of Kolmogorov-Petrovskil-Piskounov, Comm. Pure Appl. Math. 28, 323-331 (1975)

[12] J. H. Merkin and D. J. Needham, Propagating reaction-diffusion waves in a simple isothermal quadratic autocatalytic chemical system, J. Engrg. Math. 23, 343-356 (1989)

[13] J. H. Merkin, D. J. Needham, and S. K. Scott, A simple model for sustained oscillations in isothermal branched-chain or autocatalytic reactions in a well stirred open system, Proc. Roy. Soc. London Ser. A 398, 81-116 (1985)

[14] E. E. Sel'kov, Self-oscillation in glycolysis. I A simple kinetic model, European J. Biochem. 4, 79-86 (1968)

[15] L. J. Slater, Confluent Hypergeometric Functions, Cambridge Univ. Press, Cambridge, 1960

[16] A. N. Zaikin and A. M. Zhabotinskii, Concentration wave propagation in a two-dimensional liquid phase self-oscillatory system, Nature 225, 535-536 (1970) 